\title{
Spectral Efficiency and Outage Performance for Hybrid D2D-Infrastructure Uplink Cooperation
}

\author{
Ahmad Abu Al Haija, Student Member, IEEE, and Mai Vu, Senior Member, IEEE
}

\begin{abstract}
We propose a time-division uplink transmission scheme that is applicable to future-generation cellular systems by introducing hybrid device-to-device (D2D) and infrastructure cooperation. Compared with existing frequency-division schemes, the proposed time-division scheme achieves the same or better spectral efficiency and outage performance with simpler signals and shorter decoding delay. These advantages come from sending a different message in each transmission block without block Markovity as in existing schemes. Using time-division, the proposed scheme divides each transmission frame into three phases with variable duration. The two user equipment units (UEs) partially exchange their information in the first two phases and then cooperatively transmit to the base station (BS) in the third phase. We further formulate the end-to-end outage probabilities, considering outages at both UEs and the BS. We analyze this outage performance in Rayleigh fading environment assuming full channel state information (CSI) at receivers and limited CSI at transmitters. Results show that user cooperation improves the achievable rate region even under half-duplex transmission. Moreover, as the received SNR increases, this uplink cooperation significantly reduces outage probabilities and achieves the full diversity order despite additional outages at the UEs. These characteristics make the proposed scheme appealing for deployment in future cellular networks.
\end{abstract}

Index Terms - Cooperative D2D, capacity analysis, outage analysis, half-duplex transmission.

\section{INTRODUCTION}

$\mathbf{T}$ HE escalating growth of wireless networks accompanied by their multimedia services motivates system designers to deploy new technologies that efficiently utilize the wireless spectrum. Since the spectral efficiency per link has been approaching the theoretical limit for legacy cellular systems including 2nd and 3rd generations (2G and 3G) [1], multiple advanced techniques are proposed for next generation cellular standards, including the Long Term Evolution (LTE) and LTEAdvanced (LTE-A), to improve network spectral efficiency.

Manuscript received March 29, 2014; revised August 19, 2014; accepted October 9, 2014. Date of publication October 27, 2014; date of current version March 6, 2015. This work was supported in part by grants from the Natural Science and Engineering Research Council of Canada (NSERC) and in part by the Fonds Quebecois de la Recherche sur la Nature et les Technologies (FQRNT). This paper was presented in part at the IEEE Global Communications Conference Device-to-Device Communication Workshop, Atlanta, GA, USA, December 9-13, 2013. The associate editor coordinating the review of this paper and approving it for publication was S. Mao.

A. Abu Al Haija is with the Department of Electrical and Computer Engineering, McGill University, Montreal, QC H3A 0E9, Canada (e-mail: ahmad. abualhaija@mail.mcgill.ca).

M. Vu is with the Department of Electrical and Computer Engineering, Tufts University, Medford, MA 02155 USA (e-mail: maivu@ece.tufts.edu).

Color versions of one or more of the figures in this paper are available online at http://ieeexplore.ieee.org.

Digital Object Identifier 10.1109/TWC.2014.2365171

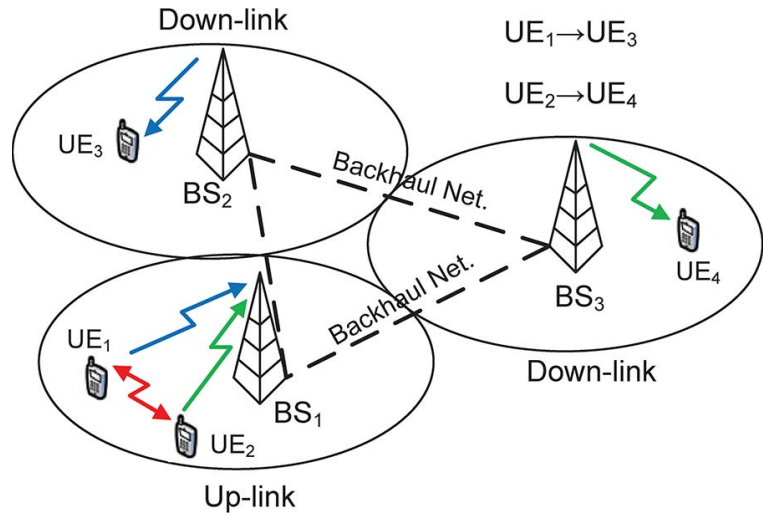

Fig. 1. D2D cooperation in uplink communication.

These techniques include multi-cell processing [2], heterogeneous network deployment and device-to-device (D2D) communication [1].

Multi-cell processing generally refers to the cooperation among different base stations (BSs) over the backhaul network connecting them to exchange the channel state information (CSI) and user data [2]. These base stations can then perform interference coordination or MIMO cooperation as in coordinated multipoint (CoMP) transmission to improve their downlink transmissions. There is little attention to over-the-air cooperation among the user-equipment in the uplink by utilizing the D2D mode. D2D allows two proximate user equipment units (UEs) to communicate directly [3]. D2D has many applications including cellular offloading [4], video dissemination [5] and smart city applications [6]. Few works, however, have considered hybrid D2D and infrastructure cooperation where the UEs utilize both the D2D mode and the infrastructure mode to cooperatively send their information to the BS. The main reason is such hybrid cooperative transmission is still not mature enough for inclusion in specific standards for practical implementation [3]. In this paper, we consider uplink user cooperation, in which two UEs cooperatively transmit to a BS, and analyze its spectral efficiency and outage performance.

\section{A. A Motivating Example}

Consider the cellular network shown in Fig. 1 where user equipment $1\left(\mathrm{UE}_{1}\right)$ wishes to communicate with $\mathrm{UE}_{3}$ and $\mathrm{UE}_{2}$ wishes to communicate with $\mathrm{UE}_{4}$. This example is valid for both homogeneous and heterogeneous networks as $\mathrm{UE}_{1}, \mathrm{UE}_{2}$ and $\mathrm{BS}_{1}$ can belong to a macro or femto cell.

In the current cellular networks and LTE-A standards [7], resource partitioning is used where each UE is given a resource 
block for its transmission to the BS. The resource blocks are orthogonal in order to reduce interference. While orthogonal transmission simplifies the signal design at UEs and the decoding at the BS, it poorly utilizes the available spectrum, which limits the achievable throughput. Here, the proximity between $\mathrm{UE}_{1}$ and $\mathrm{UE}_{2}$ may lead to strong channel links between the UEs. Hence, adding a D2D phase appears as a valuable technique for the two UEs to cooperate in order to improve their throughput to $\mathrm{BS}_{1}$. Different from pure D2D where one UE aims to send information to another UE, in hybrid cooperative transmission, the two UEs have different final destinations but choose to cooperate to help each other send information to the $\mathrm{BS}$.

Instead of resource partitioning, these UEs can cooperatively transmit to the BS in the same resource blocks, provided that they have exchanged their information beforehand. Such cooperation can be carried out with advanced signal processing at the UEs and BS and can significantly improve the spectral efficiency and outage performance, even when the resource blocks spent for information exchange between the UEs are taken into account. Existing results have shown that spectral efficiency can be improved with concurrent transmission where $\mathrm{UE}_{1}$ and $\mathrm{UE}_{2}$ transmit concurrently using the whole band and the BS decodes using successive interference cancelation (SIC) as in the multiple access channel (MAC) [8]. The spectral efficiency can be further improved when $\mathrm{UE}_{1}$ and $\mathrm{UE}_{2}$ cooperate to send their information to the $\mathrm{BS}$ by exchanging their information and perform coherent transmission (beamforming) to the BS. Such cooperative transmission requires advanced processing at the UEs and the BS as rate splitting and superposition coding are required at the UEs while joint decoding is required at the BS [9], [10]. Thanks to modern computational capability, these advanced processing now appears feasible for upcoming cellular systems. In this paper, we will show that such hybrid D2D-infrastructure cooperation can improve not only the spectral efficiency but also the reliability performance in wireless fading channels.

\section{B. Related Works}

In [11], a cooperative channel is first modeled as a multiple access channel with generalized feedback (MAC-GF) and a full-duplex information-theoretic coding scheme is proposed. This scheme has block Markov signaling, where the transmit information in two consecutive blocks is correlated, and employs backward decoding, where the BS starts decoding from the last block. This scheme is adapted to half-duplex transmission using code-division multiple-access (CDMA) in [9], FDMA in [12] and orthogonal FDMA (OFDMA) in [10]. The schemes in [9] and [10] have long delay because of backward decoding while the scheme in [12] has one block delay because of sliding window decoding but has a smaller rate region than [10] because of the specific implementation. In the CDMA scheme, generating orthogonal codes becomes more complicated for a large number of users.

Whereas existing works on cooperative transmission have been focusing on a frequency-division duplexing (FDD) implementation [10], [12], this paper analyzes a time-division duplexing (TDD) alternative and show that the TDD implementation can achieve the same or better spectral efficiency as the FDD implementation while having simpler transmit signals and shorter decoding delay. In this paper, similar to [9], [10], and [12], we consider two UEs as a basic unit of cooperation. It should be noted, however, that extension to $N$ UEs in the uplink transmission is also possible, where groups of UE pairs or all UEs in proximity to each other cooperate to send their information. We show, however, that the throughput gain diminishes with large $N$.

Further to analyzing the spectral efficiency, we also analyze the outage performance of the proposed cooperative scheme. Outage performance has not been considered in the literature for either cooperative TDD or FDD implementation. So far, outage has only been considered for the non-cooperative settings. For the non-cooperative MAC, there exist individual and common outages as defined in [13]. Assuming CSI at the transmitters, the optimal power allocations are derived to minimize the outage capacity. In [14], closed form expressions are derived for the common and individual outages of the two-user MAC assuming no CSI at the transmitters. The diversity gain region is defined in [15] and derived for the MIMO fading broadcast channel and the MAC using an error exponent analysis in [16]. The outage probabilities for different relaying techniques in the relay channel have been studied in [17] and [18]. No results so far exist, however, on outage for cooperative multiple access transmission. In this paper, we analyze the outage performance of the proposed cooperative TDD transmission. Since there is no outage performance available for exiting cooperative FDD schemes, we also extend our analysis to these schemes in order to compare the outage performance.

\section{Main Results and Contribution}

In this paper, we propose a TDD hybrid D2D-infrastructure cooperative transmission scheme for uplink multiple access communication that can be applied in future cellular systems, derive its achievable rate region and analyze its outage performance over Rayleigh fading channel. Comparing with the FDD schemes in [10] and [12], the proposed scheme has the same or better rate region, and better outage performance with simpler signal design and shorter decoding delay. This work is different from our previous work [19], in which we optimized the power allocation for maximum spectral efficiency of a fixed channel, but did not show the ML decoding analysis [20] nor consider fading channels and outage analysis. Comparing with our previous scheme in [21], the proposed scheme here achieves the same rate region but is simpler as it has less rate splitting for each UE information.

The proposed scheme sends independent information in each transmission block such that decoding immediately at the end of each block is possible. To satisfy the half-duplex constraint, each transmission block is divided through time division into 3 phases. The first two phases are for information exchange between the two UEs, and the last phase is for cooperative transmission to the $\mathrm{BS}$. While the $\mathrm{BS}$ is always in the receive mode, the two UEs alternatively transmit and receive during the first 2 phases and coherently transmit during the last phase. 
The decoding at the BS is performed using joint maximumlikelihood (ML) receiver among all the 3 phases.

We consider a single antenna at both UEs and the BS but the results can be extended to the MIMO case in a straightforward manner, which can be done in a future work. We consider block fading channel where all links remain constant over each transmission block and independently vary in the next block [22]. We assume full CSI at the receiver side with limited CSI at the transmitter side where, as in [9], each UE knows the phase of its channel to the BS such that the two UEs can employ coherent transmission. Moreover, each UE knows the relative orders between each cooperative link and the corresponding direct link so that the UE can choose it to cooperate when its cooperative link is stronger than the direct link.

We formulate and analyze both common and individual outages and extend our results in [23] by comparing with existing resource partitioning and FDD schemes. The individual outage pertains to incorrect decoding of one user information regardless of the other user information, while the common outage pertains to incorrect decoding of either user information or both. Because of the information exchanging phases, outage analysis must also consider outages at the UEs. The rate splitting and superposition coding structure also complicate outage analysis and require dependent analysis of the outage for different information parts. We further derive the outage probabilities for existing FDD implementations in [10] and [12] and compare with our TDD implementation. Results show that as the received SNR increases, the proposed TDD cooperation improves outage performance over both resource partitioning and concurrent non-cooperative transmission schemes despite additional outages at the UEs. To the best of our knowledge, ours is the first work that formulates and analyzes the outage performance for cooperative transmission with rate splitting.

\section{Paper Outline}

The rest of this paper is organized as follows. Section II describes the channel model. Section III describes the proposed TDD cooperative transmission and shows its achievable rate region and the outer bound. Section IV formulates and analyzes the common and individual outage probabilities of the proposed scheme. Section VI formulates the outage probability of existing FDD implementations and compares their performance. Section V generalizes the proposed scheme to the $N$-UE case. Section VII presents numerical results and Section VIII concludes the paper.

\section{Channel Model}

Consider the uplink communication in Fig. 1 where $\mathrm{UE}_{1}$ and $\mathrm{UE}_{2}$ wish to send their information to $\mathrm{BS}_{1}$. In the current LTE-A standard, $\mathrm{BS}_{1}$ employs resource partitioning and gives orthogonal resource blocks to the UEs for interference free transmission as shown in Fig. 2. However, when $\mathrm{UE}_{1}$ and $\mathrm{UE}_{2}$ cooperate to send their information at higher rates, the channel is quite similar to the user cooperative diversity channel defined in [9]. Hence, $\mathrm{BS}_{1}$ shall change its resource allocation to facilitate cooperation and to meet the half-duplex constraint

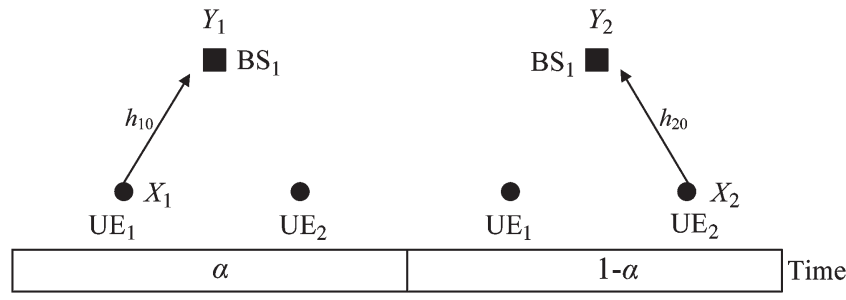

Fig. 2. Resource partitioning in current LTE standard.

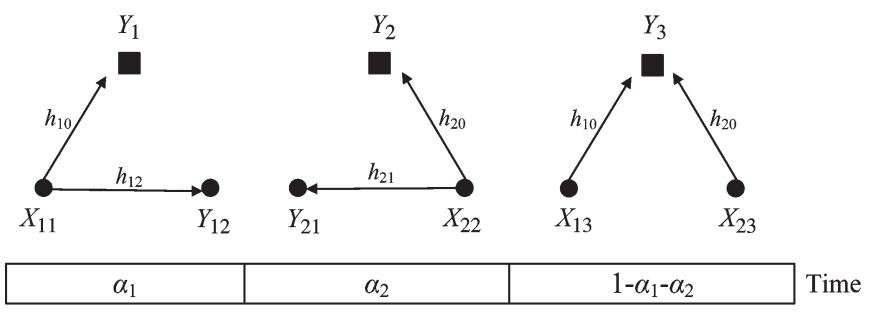

Fig. 3. Cooperative uplink transmission.

in wireless communication where each UE can only be in either the transmit or receive mode but not both at the same time and frequency band.

The proposed transmission scheme uses time division (TDD) to satisfy the half-duplex constraint. Instead of dividing the resource block into 2 orthogonal phases, $\mathrm{BS}_{1}$ divides the full resource block of $n$ symbols length into 3 phases with variable durations $\alpha_{1} n, \alpha_{2} n$ and $\left(1-\alpha_{1}-\alpha_{2}\right) n$ as shown in Fig. 3 . While $\mathrm{BS}_{1}$ is always in receiving mode, each UE either transmits or receives during the first two phases and both transmit during the 3rd phase. We consider a single antenna at each UE and the BS but the scheme can be extended to the MIMO case. Then, the discrete-time channel model for the half-duplex uplink transmission can be expressed in each phase as follows.

$$
\begin{aligned}
& \text { phase 1: } Y_{12}=h_{12} X_{11}+Z_{12}, \quad Y_{1}=h_{10} X_{11}+Z_{1}, \\
& \text { phase 2: } Y_{21}=h_{21} X_{22}+Z_{21}, \quad Y_{2}=h_{20} X_{22}+Z_{2}, \\
& \text { phase 3: } Y_{3}=h_{10} X_{13}+h_{20} X_{23}+Z_{3},
\end{aligned}
$$

where $Y_{i j},(i, j) \in\{1,2\}$, is the signal received by the $j$ th $\mathrm{UE}$ during the $i$ th phase; $Y_{k}, k \in\{1,2,3\}$ is the signal received by $\mathrm{BS}_{1}$ during the $k$ th phase; and all the $Z_{l}, l \in\{12,21,1,2,3\}$, are i.i.d complex Gaussian noises with zero mean and unit variance. $X_{11}$ and $X_{13}$ are the signals transmitted from $\mathrm{UE}_{1}$ during the 1 st and 3rd phases, respectively. Similarly, $X_{22}$ and $X_{23}$ are the signals transmitted from $\mathrm{UE}_{2}$ during the 2 nd and 3rd phases.

Each link coefficient is affected by Rayleigh fading and path loss as follows.

$$
h=\tilde{h} /\left(d^{\gamma / 2}\right)
$$

where $\tilde{h}$ is the small scale fading component and has a complex Gaussian distribution with zero mean and unit variance $(\mathcal{N}(0,1))$. The large scale fading component is captured by a path loss model where $d$ is the distance between two nodes in the network and $\gamma$ is the attenuation factor. Let $g=|h|$ and $\theta$ be the amplitude and the phase of a link coefficient, then $g$ has Rayleigh distribution while $\theta$ has uniform distribution in the interval $[0,2 \pi]$. 
We assume full CSI at receivers, i.e., $\mathrm{BS}_{1}$ knows $h_{10}$ and $h_{20}, \mathrm{UE}_{1}$ knows $h_{21}$ and $\mathrm{UE}_{2}$ knows $h_{12}$. This receiver CSI is a standard assumption and can be obtained directly via channel estimation. We also assume partial CSI at transmitters where each UE knows the phase of its link to the BS and the relative amplitude orders between each cooperative link and the corresponding direct link. The phase knowledge allows coherent transmission between the UEs to obtain the beamforming gain to the BS and is a standard assumption in coherent relaying literature [9], [10], [24]. Here we distinguish between CSI at receivers and transmitters, but a node (such as a UE) can act as both a transmitter and a receiver depending on the transmission phase. The transmitter CSI can be obtained via the reciprocity between forward and reverse channels as in a time-division duplexed (TDDD) system, or via feedback from the BS. For example, $\mathrm{UE}_{1}$ can send the amplitude information of the $h_{21}$ link $\left(g_{21}\right)$ to the BS and similarly, $\mathrm{UE}_{2}$ sends the amplitude information $g_{12}$ to the BS. Then, the BS compares these amplitudes, $g_{12}$ with $g_{10}$ and $g_{21}$ with $g_{20}$, and feeds back to both UEs 2-bit information to tell the UEs about the link orders $\left(g_{12} \gtrless g_{10}\right.$ and $\left.g_{21} \gtrless g_{20}\right)$. The link order knowledge is important for the UEs in order to avoid cooperation when the cooperative links are weak since then decoding at the UEs reduces the achievable rate region. The best transmission scenario for each links order is shown in Section III-D.

We assume block fading where the channel coefficients stay constant in each block through all 3 phases and change independently in the next block. Furthermore, we assume here perfect infinite precision CSI, where available, as we aim to study the performance limits of the proposed scheme. Additional analyses are required when the CSI is quantized, including the phases sent from the BS to the UEs and the link amplitudes $\left(g_{12}, g_{21}\right)$ sent from the UEs to the BS. Quantized phase knowledge will impact the coherent transmission as the signals from the UEs will not arrive completely in phase at the BS. Quantized link amplitudes will impact the optimal transmission scenario decided by the BS that compares the direct link amplitudes with quantized cooperative link amplitudes. Understanding these effects of quantized CSI is important and can be considered in a future work.

Note that this model can be applied to OFDDMA systems, such as LTE networks [7], where a number of resource blocks is allocated for each UE in the time-frequency grid. In order to adapt our scheme into an OFDDMA system, during the hybrid D2D-infrastructure mode, the BS can allocate different resource blocks for each UE in the first two phases (specifically blocks in the same frequency but at different times to fit with the proposed scheme). However, in the third phase, the BS will allocate the same resource blocks for both UEs to allow them to perform coherent transmission.

\section{A Time-Division Uplink CoOperative TRANSMISSION SCHEME}

In this section, we describe the proposed TDD cooperative scheme applied to the half-duplex uplink communication in LTE-A networks. We establish its theoretical achievable rate re-

\begin{tabular}{|c|c|c|c|}
\hline Time & $\alpha_{1}$ & $\alpha_{2}$ & $1-\alpha_{1}-\alpha_{2}$ \\
\hline $\mathrm{UE}_{1}$ & $X_{11}(i)$ & $Y_{21} \rightarrow \widetilde{k}$ & $X_{13}(j, i, \tilde{k})$ \\
\hline $\mathrm{UE}_{2}$ & $Y_{12} \rightarrow \widetilde{i}$ & $X_{22}(k)$ & $X_{23}(l, \tilde{i}, k)$ \\
\hline \multirow{2}{*}{ BS } & $\overline{Y_{1}}$ & $Y_{2}$ & $Y_{3}$ \\
\hline & \multicolumn{3}{|c|}{$(\hat{j}, \hat{l}, \hat{i}, \hat{k})$} \\
\hline
\end{tabular}

Fig. 4. Cooperative uplink transmission scheme (light shade $=$ transmit signal, dark shade $=$ received signal and decoded indices).

gion based on rigorous ML decoding analysis. We also present an outer bound and compare it to the achievable rate region.

Compared with the schemes in [9] and [11], the proposed scheme has better spectral efficiency, simpler signal design and shorter decoding delay (no block delay). These characteristics apply since the two UEs encode independent information in each transmission block and the BS decodes directly at the end of each block instead of backward decoding. The proposed scheme is based on rate splitting, superposition coding and partial decode-forward (PDF) relaying techniques.

The transmission in each block is divided into three phases with relative durations $\alpha_{1}, \alpha_{2}$ and $\alpha_{3}=1-\alpha_{1}-\alpha_{2}$ (see Fig. 4). In each block, $\mathrm{UE}_{1}$ splits its information into two parts: a cooperative part with index $i$ and a private part with index $j$. It sends the private part directly to the $\mathrm{BS}$ at rate $R_{10}$ and sends the cooperative part to the $\mathrm{BS}$ in cooperation with $\mathrm{UE}_{2}$ at rate $R_{12}$. These parts are then encoded using superposition coding, in which for each transmit sequence of a cooperative part, a group of sequences is generated for the private parts. Similarly, $\mathrm{UE}_{2}$ splits its information into a cooperative part (indexed by $k$ ) and a private part (indexed by $l$ ) and encodes them using superposition coding. In the first two phases, the two UEs exchange the cooperative parts. In the 3rd phase, each UE sends both cooperative information parts and its own private part to the BS. Effectively each UE performs PDF relaying of the cooperative part of the other UE. Next, we describe in detail the control signaling, transmit signals and ML receiver with help from Fig. 4.

\section{A. Control Signaling}

The D2D connection set-up includes the peer discovery, paging and connection establishment [3]. In a 3GPP network, the peer discovery can be performed by two approaches. The first approach is called a centralized approach where the Mobility Management Entity (MME) detects two UEs who can setup a D2D communication to improve the throughput. Then, the MME asks the BS to request measurements from these UEs and compare between the direct and cooperative links amplitudes. After that, the BS sends a feedback to the UEs telling them to switch to the D2D cooperative mode with the specific transmission scenario as one of the four cases described in Section III-D. The second approach is called a distributed approach where each UE broadcasts its identity such that other UEs are aware of its existence. If two UEs are close to each other, they can request the BS for cooperative transmission. Then, the BS performs the measurements of the cooperative and direct link amplitudes and sends feedback to the UEs informing them about the optimal transmission scenario. 


\section{B. Transmit Signals}

1) Transmit Sequences Generation: As in all communication systems, the channel encoder maps each piece of input information into a unique sequence. This sequence includes some controlled redundancy of the input information that can be used by the receiver to alleviate the noise encountered during transmission to reduce decoding error.

Let $\mathcal{I}(\mathcal{K})$ and $\mathcal{J}(\mathcal{L})$ be the sets of signal indices for the cooperative and private parts of $\mathrm{UE}_{1}\left(\mathrm{UE}_{2}\right)$, respectively. Since the transmission is affected by Gaussian noise as in (1), both UEs employ Gaussian signaling to maximize the transmission rate [24]. The Gaussian signals are generated as follows. For each element $i \in \mathcal{I}$, independently generate a signal vector (sequence) $\boldsymbol{u}_{1, i}$ of length $n$ according to a Gaussian distribution with zero mean and unit variance. This sequence is scaled by a power from $\mathrm{UE}_{1}$ as shown in Section III-B2. Similar and independent Gaussian sequences $\boldsymbol{u}_{2, k}$ and $\boldsymbol{u}_{3, i, k}$ are generated for each element $k \in \mathcal{K}$ and each pair $(i, k)$, respectively. Next, perform superposition signaling where for each sequence $\boldsymbol{u}_{3, i, k}$, generate a Gaussian sequence $\boldsymbol{x}_{13, j, i, k}\left(\boldsymbol{x}_{23, l, i, k}\right)$ for each $j \in \mathcal{J}(l \in \mathcal{L})$. The superposition coding reduces the decoding complexity and increases the rate region as shown in Section III-C.

2) Transmission Scheme: In the 1st phase, $\mathrm{UE}_{1}$ sends its cooperative information at rate $R_{12}$ by transmitting the signal $X_{11, i}$ that consists of the first $\alpha_{1} n$ elements of a scaled sequence of $\boldsymbol{u}_{1, i}$ as shown in (3). By the end of the 1st phase, $\mathrm{UE}_{2}$ decodes $X_{11, i}$. Then, in the $3 \mathrm{rd}$ phase, $\mathrm{UE}_{1}$ sends its private information and both cooperative information parts at rate triplet $\left(R_{10}, R_{12}, R_{21}\right)$ by transmitting the signal $X_{13, j, i, k}$, which consists of the last $\alpha_{3} n$ elements of sequence $\boldsymbol{x}_{13, j, i, k}$. Similarly, $\mathrm{UE}_{2}$ transmit the signals $X_{22, k}$ and $X_{23, l, i, k}$ in the 2 nd and 3rd, respectively. Since both UEs know indices $i$ and $k$ in this phase, they can perform coherent transmission of these cooperative information by transmit beamforming such that the achievable rates of both UEs are increased. The transmit signals at each phase are

$$
\begin{aligned}
\text { phase 1: } & X_{11, i}=\sqrt{\rho_{11}} U_{1}(i), \\
\text { phase 2: } & X_{22, k}=\sqrt{\rho_{22}} U_{2}(k), \\
\text { phase 3: } & X_{13, j, i, k}=\sqrt{\rho_{10}} V_{1}(j)+\sqrt{\rho_{13}} U_{3}(i, k), \\
& X_{23, l, i, k}=\sqrt{\rho_{20}} V_{2}(l)+\sqrt{\rho_{23}} U_{3}(i, k)
\end{aligned}
$$

where $U_{1}, U_{2}, V_{1}, V_{2}$, and $U_{3}$ are independent and identically distributed Gaussian signals with zero mean and unit variance, $X_{13}$ and $X_{23}$ are superpositioned on $U_{3}$. Here, $\rho_{11}, \rho_{22}, \rho_{10}$, and $\rho_{20}$ are the transmission powers allocated for signals $U_{1}, U_{2}, V_{1}$ and $V_{2}$, respectively, $\rho_{13}$ and $\rho_{23}$ are the transmission powers allocated for signal $U_{3}$ by $\mathrm{UE}_{1}$ and $\mathrm{UE}_{2}$, respectively. Let $\mathcal{P}_{1}$ and $\mathcal{P}_{2}$ be the total transmission power for $\mathrm{UE}_{1}$ and $\mathrm{UE}_{2}$, respectively. Then, we have the following power constraints:

$$
\begin{aligned}
& \alpha_{1} \rho_{11}+\alpha_{3}\left(\rho_{10}+\rho_{13}\right)=\mathcal{P}_{1}, \\
& \alpha_{2} \rho_{22}+\alpha_{3}\left(\rho_{20}+\rho_{23}\right)=\mathcal{P}_{2} .
\end{aligned}
$$

\section{ML Receiver}

Assume that all sequences in any set $\mathcal{I}, \mathcal{J}, \mathcal{K}$, or $\mathcal{L}$, have equal transmission probability. Sequence maximum likelihood (ML) criterion is then optimal and achieves the same performance as the optimal maximum a posterior probability (MAP) criterion.

At Each UE: In the 1st phase, $\mathrm{UE}_{2}$ detects $i$ from $Y_{12}$ using sequence ML criterion. Hence, for a given sequence $\boldsymbol{y}_{12}$ of length $\alpha_{1} n, \mathrm{UE}_{2}$ chooses $\hat{x}_{11, \hat{i}}$ to be the transmitted sequence if

$$
p\left(\boldsymbol{y}_{12} \mid \hat{\boldsymbol{x}}_{11, \hat{i}}\right) \geq p\left(\boldsymbol{y}_{12} \mid \boldsymbol{x}_{11, i}\right), \text { for all } \boldsymbol{x}_{11}(i) \neq \hat{\boldsymbol{x}}_{11}(\hat{i}) .
$$

$\mathrm{UE}_{1}$ applies similar decoding rule in the 2nd phase. Hence, $\mathrm{UE}_{1}$ and $\mathrm{UE}_{2}$ can reliably detect the transmit sequences $\boldsymbol{x}_{11, i}$ and $\boldsymbol{x}_{22, k}$, respectively, if

$$
\begin{aligned}
R_{12} & \leq \alpha_{1} \log \left(1+g_{12}^{2} \rho_{11}\right)=J_{1} \\
\text { and } R_{21} & \leq \alpha_{2} \log \left(1+g_{21}^{2} \rho_{22}\right)=J_{2} .
\end{aligned}
$$

At the Base Station: The BS utilizes the received signals in all three phases $\left(Y_{1}, Y_{2}, Y_{3}\right)$ to jointly detect all information parts $(j, l, i, k)$ using joint sequence ML criterion. With the signals in (3), the received signals at the BS are given as follows.

Phase 1: $Y_{1}=h_{10} \sqrt{\rho_{11}} U_{1}(i)+Z_{1}$,

Phase 2: $Y_{2}=h_{20} \sqrt{\rho_{11}} U_{2}(k)+Z_{2}$,

Phase 3: $Y_{3}=h_{10} \sqrt{\rho_{10}} V_{1}(j)+h_{20} \sqrt{\rho_{20}} V_{2}(l)$

$$
+\left(h_{10} \sqrt{\rho_{13}}+h_{20} \sqrt{\rho_{23}}\right) U_{3}(i, k)+Z_{3} .
$$

Then, for given received sequences $\boldsymbol{y}_{1}$ of length $\alpha_{1} n, \boldsymbol{y}_{2}$ of length $\alpha_{2} n$ and $\boldsymbol{y}_{3}$ of length $\alpha_{3} n$, the BS chooses $\hat{\boldsymbol{x}}_{11, \hat{i}}, \hat{\boldsymbol{x}}_{22, \hat{k}}$, $\hat{\boldsymbol{x}}_{10, \hat{j}, \hat{i}, \hat{k}}$ and $\hat{\boldsymbol{x}}_{20, \hat{l}, \hat{i}, \hat{k}}$ to be the transmitted sequences if:

$$
\begin{aligned}
& P\left(\boldsymbol{y}_{1} \mid \hat{\boldsymbol{x}}_{11, \hat{i}}\right) P\left(\boldsymbol{y}_{2} \mid \hat{\boldsymbol{x}}_{22, \hat{k}}\right) P\left(\boldsymbol{y}_{3} \mid \hat{\boldsymbol{x}}_{10, \hat{j}, \hat{i}, \hat{k}}, \hat{\boldsymbol{x}}_{20, \hat{l}, \hat{i}, \hat{k}}\right) \geq \\
& P\left(\boldsymbol{y}_{1} \mid \boldsymbol{x}_{11, i}\right) P\left(\boldsymbol{y}_{2} \mid \boldsymbol{x}_{22, k}\right) P\left(\boldsymbol{y}_{3} \mid \boldsymbol{x}_{10, j, i, k}, \boldsymbol{x}_{20, l, i, k}\right) \\
& \text { for all } \boldsymbol{x}_{11, i} \neq \hat{\boldsymbol{x}}_{11, \hat{i}}, \boldsymbol{x}_{22, k} \neq \hat{\boldsymbol{x}}_{22, \hat{k}}, \boldsymbol{x}_{10, j, i, k} \neq \hat{\boldsymbol{x}}_{10, \hat{j}, \hat{i}, \hat{k}} \\
& \text { and } \boldsymbol{x}_{20, l, i, k} \neq \hat{\boldsymbol{x}}_{20, \hat{l}, \hat{i}, \hat{k}} .
\end{aligned}
$$

Lemma 1: For each channel realization, the rate constraints that ensure vanishing decoding error probabilities at the BS are given as

$$
\begin{gathered}
R_{10} \leq \alpha_{3} \log \left(1+g_{10}^{2} \rho_{10}\right)=J_{3}, \\
R_{20} \leq \alpha_{3} \log \left(1+g_{20}^{2} \rho_{20}\right)=J_{4}, \\
R_{10}+R_{20} \leq \alpha_{3} \log \left(1+g_{10}^{2} \rho_{10}+g_{20}^{2} \rho_{20}\right)=J_{5} \\
R_{1}+R_{20} \leq \alpha_{1} \log \left(1+g_{10}^{2} \rho_{11}\right)+\alpha_{3} \zeta=J_{6}, \\
R_{10}+R_{2} \leq \alpha_{2} \log \left(1+g_{20}^{2} \rho_{22}\right)+\alpha_{3} \zeta=J_{7} \\
R_{1}+R_{2} \leq \alpha_{1} \log \left(1+g_{10}^{2} \rho_{11}\right) \\
\quad+\alpha_{2} \log \left(1+g_{20}^{2} \rho_{22}\right)+\alpha_{3} \zeta=J_{8}, \\
\zeta=\log \left(1+g_{10}^{2} \rho_{10}+g_{20}^{2} \rho_{20}\right. \\
\left.+\left(g_{10} \sqrt{\rho_{13}}+g_{20} \sqrt{\rho_{23}}\right)^{2}\right) .
\end{gathered}
$$


Hence, the BS can reliably decode all information parts if (9) is satisfied. Note that $\zeta$ shows the advantage of beamforming resulted from coherent transmission of $(i, k)$ from both UEs in the 3rd phase.

Sketch of the proof: A decoding error can occur for the cooperative or the private parts or both. However, because of superposition coding, if either cooperative part is incorrectly decoded, both private parts will also be decoded incorrectly. Hence, we consider two cases:

1) The cooperative parts are decoded correctly:

When both cooperative parts have been decoded correctly, the BS can decode the private parts from $Y_{3}$ after removing $U_{3}(i, k)$. Then, $Y_{3}$ becomes similar to the received signal in a MAC. Hence, the rate constraints for private parts are similar to those of a MAC as given by $J_{3}, J_{4}$, and $J_{5}$ in (9).

2) Either cooperative part or both are decoded incorrectly: This case contains three sub-cases: only one of the two cooperative parts is decoded correctly, or both are decoded incorrectly, each of which leads to a different rate constraint. If the BS decodes $i$ incorrectly but decodes $k$ correctly, then both $j$ and $l$ will be decoded incorrectly. Because of the joint decoding performed at the BS as in (8), this incorrect decoding will result in a constraint on the total rate of the parts $i, j$ and $l$. Since $i$ is sent in phases 1 and 3, this rate constraint is obtained from $Y_{1}$ and $Y_{3}$ as follows:

$$
\begin{aligned}
R_{12}+R_{10}+R_{20} & \leq \alpha_{1} \log \left(1+\mathrm{SNR}_{1}\right) \\
& +\alpha_{3} \log \left(1+\mathrm{SNR}_{3}\right),
\end{aligned}
$$

where $\mathrm{SNR}_{t}, t \in\{1,2,3\}$ is the SNR of all the received signals (due to BS joint decoding) in phase $t$. Thus (10) reduces to $J_{6}$ in (9) where $\mathrm{SNR}_{3}$ is obtained over all incorrectly decoded signal parts $\left(V_{1}, V_{2}, U_{3}\right)$ in $Y_{3}$. Similarly, we can obtain $J_{7}$ if the BS decodes $k$ incorrectly but decodes $i$ correctly. If the BS decodes both $i$ and $k$ incorrectly, then all messages parts $i, k, j$ and $l$ will be in error and we obtain the following rate constraint:

$$
\begin{aligned}
R_{12}+R_{10} & +R_{21}+R_{20} \leq \alpha_{1} \log \left(1+\mathrm{SNR}_{1}\right) \\
& +\alpha_{2} \log \left(1+\mathrm{SNR}_{2}\right)+\alpha_{3} \log \left(1+\mathrm{SNR}_{3}\right),
\end{aligned}
$$

which results in constraint $J_{8}$ in (9).

A full analysis based on ML decoding can be found in Appendix A.

We note that the achievable rate region in (9) is a direct result of the joint ML decoding performed at the BS simultaneously over all three phases as in (8). If the BS uses sequential decoding or decodes each phase separately, this can reduce the decoding complexity but will result in a strictly smaller rate region.

\section{Achievable Rate Region and Transmission Scenarios}

The achievable rate region in terms of $R_{1}=R_{10}+R_{12}$ and $R_{2}=R_{20}+R_{21}$ is given as follows.

Theorem 1: The achievable rate region resulting from the proposed scheme for each channel realization consists of the union of all rate pairs $\left(R_{1}, R_{2}\right)$ satisfying the following constraints:

$$
\begin{gathered}
R_{1} \leq J_{1}+J_{3}, \quad R_{2} \leq J_{2}+J_{4} \\
R_{1}+R_{2} \leq J_{1}+J_{2}+J_{5}, \quad R_{1}+R_{2} \leq J_{8}
\end{gathered}
$$

for some $\alpha_{1} \geq 0, \alpha_{2} \geq 0, \alpha_{1}+\alpha_{2} \leq 1$ and power allocation set $\left(\rho_{10}, \rho_{20}, \rho_{11}, \rho_{22}, \rho_{13}, \rho_{23}\right)$ satisfying (4) where $J_{1}-J_{8}$ are given in (6) and (9).

Proof: Obtained by combining (6) and (9). See Appendix A for more details.

Combining (6) and (9) leads to the constraints in (12) and 2 other constraints as $R_{1}+R_{2} \leq J_{1}+J_{7}$ and $R_{1}+R_{2} \leq J_{2}+J_{6}$. However, these constraints are redundant as stated below.

Corollary 1: Two sum rate constraints $\left(R_{1}+R_{2} \leq \min \left\{\left(J_{1}+\right.\right.\right.$ $\left.\left.J_{7}, J_{2}+J_{6}\right)\right\}$ ) on the achievable region result from combining (6) and (9) are redundant.

Proof: For any channel configuration, $\min \left\{\left(J_{1}+J_{7}\right.\right.$, $\left.\left.\left.\left.J_{2}+J_{6}\right)\right\}\right) \geq \min \left\{\left(J_{1}+J_{2}+J_{5}, J_{8}\right)\right\}\right)$.

From the proposed scheme, 4 optimal sub-schemes can be obtained depending on the channel configuration. These schemes have different power allocation and phase durations that are results of the operating scenario. Each UE requires only the relative amplitude orders between each cooperative link and the corresponding direct link to determine which scenario to operate. Since the BS knows all links as discussed in Section II and there are 2 pairs of links, this knowledge can be obtained through a 2-bit feedback from the BS which incurs negligible overhead. Each bit indicates the relation between one pair of direct and cooperative links. Assume that at the beginning of each transmission block, the UEs have sufficient knowledge of the link orders, the operating scenarios are given as follows.

1) Case 1 ( $g_{12} \leq g_{10}$ and $\left.g_{21} \leq g_{20}\right)$, Direct Transmissions for Both UEs: In this case, decoding at the two UEs actually limits the achievable rates because the inter-UE links are weaker than the direct links. Therefore, both UEs transmit directly to the BS all the time without cooperation as in the concurrent transmission with SIC. The achievable rate is given in (12) but with $\alpha_{1}=\alpha_{2}=0, \rho_{11}=\rho_{13}=\rho_{22}=\rho_{23}=0$, $\rho_{10}=\mathcal{P}_{1}$ and $\rho_{20}=\mathcal{P}_{2}$.

2) Case $2\left(g_{12}>g_{10}\right.$ and $\left.g_{21}>g_{20}\right)$, Cooperation for Both UEs: In this case, both UEs obtain mutual benefit from cooperation for sending their information to the BS. When $g_{12}>g_{10}$ and $g_{21}>g_{20}, J_{2}+J_{6}>J_{8}$, and $J_{1}+J_{7}>J_{8}$. Therefore, the rate constraints are as in (12) with all signals and phases.

3) Case $3\left(g_{12}>g_{10}\right.$ and $\left.g_{21} \leq g_{20}\right)$, Cooperation for $U E_{1}$ and Direct Transmission for $U_{2}$ : Here, $\mathrm{UE}_{1}$ prefers cooperation while $\mathrm{UE}_{2}$ prefers direct transmission. Therefore, the transmission is carried over 2 phases only where $\mathrm{UE}_{2}$ relays information for $\mathrm{UE}_{1}$ while also sending its own information. $\mathrm{UE}_{1}$ sends its cooperative part in phase 1 . In phase $2, \mathrm{UE}_{1}$ sends its two parts while $\mathrm{UE}_{2}$ sends its full information and the cooperative part of $\mathrm{UE}_{1}$. The achievable rate is given in (12) with $\alpha_{2}=0$, and $\rho_{22}=0$.

4) Case $4\left(g_{12} \leq g_{10}\right.$ and $\left.g_{21}>g_{20}\right)$, Cooperation for $U E_{2}$ and Direct Transmission for $U E_{1}$ : This case is the opposite of the Case 3 where the achievable rate is given in (12) with $\alpha_{1}=0$, and $\rho_{11}=0$. 


\section{E. Outer Bound}

In this section, we provide an outer bound with constraints similar to that in Theorem 1. During the 3rd phase, the channel looks like a MAC with common message [25] while during the first two phases, it looks like a broadcast channel (BC). Furthermore, when one UE has no information to send, the channel becomes as the relay channel (RC). Although capacity is known for the MAC with common message and for the Gaussian BC, the capacity for RC is unknown in general. In [26], an outer bound is derived for the full-duplex scheme in [9] based on the idea of dependence balance [27]. When applied to the proposed half-duplex transmission, the outer bound holds without dependence balance condition as follows.

Corollary 2. [26]: An outer bound for the uplink halfduplex D2D communication consists of all rate pairs $\left(R_{1}, R_{2}\right)$ satisfying (12) but replacing $g_{12}^{2}\left(g_{21}^{2}\right)$ by $g_{12}^{2}+g_{10}^{2}\left(g_{21}^{2}+g_{20}^{2}\right)$ as follows.

$$
\begin{aligned}
R_{1} \leq & \alpha_{1} \log \left(1+\left(g_{10}^{2}+g_{12}^{2}\right) \rho_{11}\right)+J_{3}, \\
R_{2} \leq & \alpha_{2} \log \left(1+\left(g_{20}^{2}+g_{21}^{2}\right) \rho_{22}\right)+J_{4}, \\
R_{1}+R_{2} \leq & \alpha_{1} \log \left(1+\left(g_{10}^{2}+g_{12}^{2}\right) \rho_{11}\right) \\
& +\alpha_{2} \log \left(1+\left(g_{20}^{2}+g_{21}^{2}\right) \rho_{22}\right)+J_{5}, \\
R_{1}+R_{2} \leq & J_{8} .
\end{aligned}
$$

MIMO View: These bounds can be obtained using MIMO bounds at receiver and transmitter sides. Consider the 1 st phase, let $\mathrm{UE}_{1}$ transmits while $\mathrm{UE}_{2}$ and the $\mathrm{BS}$ receive with full cooperation as in a SIMO $(1 \times 2)$ channel, this gives the first outer bound on $R_{1}$ in (13). The second bound (on $R_{2}$ ) is obtained in a similar way. For the third bound (on $R_{1}+R_{2}$ ), the 1 st and 2 nd phases are bounded using a SIMO, similarly to that for $R_{1}$ and $R_{2}$, respectively; in the 3rd phase, since we use the MAC bound at the receiver side, where both UEs transmit without cooperation, which results in the term $J_{5}$. Finally, the fourth bound (on $R_{1}+R_{2}$ ) is obtained from the MISO bound at the transmitter side: in the 1 st phase, only $\mathrm{UE}_{1}$ sends and the $\mathrm{BS}$ receives given known signal from $\mathrm{UE}_{2}$; the same holds for the 2nd phase; in the 3rd phase, both UEs transmit with full cooperation as in a MISO $(2 \times 1)$ channel.

Note that the tightness of the outer bound is determined by the ratios $g_{12}^{2} / g_{10}^{2}$ and $g_{21}^{2} / g_{20}^{2}$. The outer bound becomes tighter as these two ratios increase since then $g_{12}^{2} \rightarrow g_{12}^{2}+g_{10}^{2}$ and $g_{21}^{2} \rightarrow g_{21}^{2}+g_{20}^{2}$. In other words, the bound becomes increasingly tight as the inter-UE link qualities increase.

\section{Outage Probability and Outage Rate Region}

The previous analysis provides the region of transmission rates that can be achieved for each fading channel realization. In most wireless services, however, a minimum target information rate is required to support the service, below which the service is unsustainable. For a particular fading realization, the channel may or may not support the target rate. The probability that the rate supported by the fading channel falls below the target rate is called the outage rate probability. Outage has been analyzed for non-cooperative concurrent transmission with SIC (classical MAC) [13], [14] but has not been formulated or analyzed in a cooperative setting.

In this section, we formulate and analyze the outage probability of the proposed cooperative scheme. Suppose that based on the service requirements, the target rate pair is $\left(R_{1}, R_{2}\right)$. Outage occurs in the event that the target rate pair lies outside the achievable region for a channel realization. There are two types of outage in multi-user transmission: common and individual outage [13], [14]. The individual outage for $\mathrm{UE}_{1}$ is the probability that the channel cannot support its transmission rate regardless of whether the channel can or cannot support the transmission rate of $\mathrm{UE}_{2}$. The common outage is the probability that the channel cannot support the transmission rate of either $\mathrm{UE}_{1}$ or $\mathrm{UE}_{2}$ or both.

Unlike the non-cooperative schemes where outage occurs only at the BS, outage in the proposed cooperative scheme can also occur at the UEs. Moreover, the outage formulation can be different for each channel configuration depending on the specific transmission scheme used for that realization as outlined in the 4 cases in Section III-D.

Define $P_{c m}, P_{1 m}$, and $P_{2 m}$ for $m \in\{1,2,3,4\}$ as the common and individual outage probabilities for case $m$ as discussed in Section III-D. Then, the outage probability is given as follows.

Theorem 2: For the proposed 3-phase scheme, the average common outage probability $\left(\bar{P}_{c}\right)$ is given as

$$
\begin{aligned}
\bar{P}_{c}= & P\left[g_{12} \leq g_{10}, g_{21} \leq g_{20}\right] P_{c 1} \\
& +P\left[g_{12}>g_{10}, g_{21}>g_{20}\right] P_{c 2} \\
& +P\left[g_{12}>g_{10}, g_{21} \leq g_{20}\right] P_{c 3} \\
& +P\left[g_{12} \leq g_{10}, g_{21}>g_{20}\right] P_{c 4}
\end{aligned}
$$

where $P_{c 1}$ and $P_{c 2}$ are given in Section IV-A and $\mathrm{B}$, respectively, while $P_{c 3}$ and $P_{c 4}$ are given in Section IV-C. The average individual outage probabilities $\left(\bar{P}_{1}, \bar{P}_{2}\right)$ have similar formulation.

Proof: Obtained by formulating the outage probability of each case as in the following sections.

\section{A. Outage Probability for Transmission Case 1}

This case occurs when $g_{12} \leq g_{10}, g_{21} \leq g_{20}$ and it is the same as the classical non-cooperative MAC. The probability for this case is obtained as follows.

Lemma 2: The probability for case 1 is given as

$$
P\left[g_{12}>g_{10}, g_{21}>g_{20}\right]=\frac{\mu_{10}}{\mu_{12}+\mu_{10}} \frac{\mu_{20}}{\mu_{21}+\mu_{20}}
$$

where $\mu_{i j}$ is the mean of $g_{i j}^{2}$ for $i \in\{1,2\}$ and $j \in\{0,1,2\}$.

Proof: See Appendix B.

The common and individual outage probabilities $\left(P_{c 1}, P_{11}, P_{21}\right)$ for this case are defined in [14]. Hence, the outage probabilities for this case are similar to that in [14] except that each outage probability is conditioned on the event that $g_{12} \leq g_{10}$ and $g_{21} \leq g_{20}$. 


\section{B. Outage Probability for Transmission Case 2}

This case applies when $g_{12}>g_{10}, g_{21}>g_{20}$, which allows full cooperation between the two UEs. The probability for this case is the same as (15) but replacing $\mu_{10}$ by $\mu_{12}$ and $\mu_{20}$ by $\mu_{21}$ in the numerator. In this case, since the two UEs perform rate splitting and partial decode-forwarding, the target rates $\left(R_{1}, R_{2}\right)$ are split into the cooperative and private target rates as described in Section III. Different from the non-cooperative MAC, here outage can occur at either UE or at the BS. We first analyze outage probabilities at the UEs and the BS separately, then combine them to obtain the overall outage probability.

1) Outage at the UEs: As $\mathrm{UE}_{1}$ has no CSI about $g_{12}$, the transmission rate $R_{12}$ may exceed $J_{1}$ in (6), which is the maximum rate supported by the fading channel to $\mathrm{UE}_{2}$. Therefore, there is a possibility for outage at $\mathrm{UE}_{2}$. The outage probability at $\mathrm{UE}_{2}\left(P_{m 2}\right)$ is given as

$$
\begin{aligned}
P_{m 2} & =P\left[\alpha_{2} \log \left(1+g_{12}^{2} \rho_{11}\right) \leq R_{12} \mid g_{12}>g_{10}, g_{21}>g_{20}\right] \\
& =P\left[g_{12}^{2} \leq \frac{2^{\alpha_{2} R_{12}}-1}{\rho_{11}} \mid g_{12}>g_{10}\right] .
\end{aligned}
$$

Similar formula holds for the outage probability at $\operatorname{UE}_{1}\left(P_{m 1}\right)$.

2) Outage at the Base Station: The outage at the BS is considered when there are no outages at the UEs. This outage is tied directly with the decoding constraints of the cooperative and private information parts as shown in (9). This outage consists of two parts, for the cooperative and the private information.

Because of the superposition coding structure that each private part is superimposed on both cooperative parts, an outage for either of the cooperative information parts leads to an outage for both private parts. Hence we only need to consider the common outage for the cooperative parts, but need to consider both the common and individual outage for the private parts.

Remark 1: For the achievable rate region in (12), we look at the combination of (6) and (9) and we show in Theorem 1 that two rate constraints $R_{1}+R_{20} \leq J_{6}$ and $R_{10}+R_{2} \leq J_{7}$ in (9) are redundant. However, in the outage analysis, we look at the outage at the UEs and the BS separately. Hence, these 2 constraints at the BS are active and they affect the outage of the cooperative parts.

3) Outage of the Cooperative Parts: From (9), the rate constraints for the cooperative parts are

$$
\begin{aligned}
R_{12} & \leq J_{6}-\left(R_{10}+R_{20}\right), \\
R_{21} & \leq J_{7}-\left(R_{10}+R_{20}\right), \\
R_{12}+R_{21} & \leq J_{8}-\left(R_{10}+R_{20}\right) .
\end{aligned}
$$

For fixed target rates $\left(R_{10}, R_{12}, R_{20}, R_{21}\right)$, a common outage of the cooperative parts occurs when the cooperative target rate pair $\left(R_{12}, R_{21}\right)$ lies outside the region obtained from (17). The probability of this cooperative common outage is given as

$$
\begin{aligned}
P_{c c}=1-P[ & R_{12} \leq J_{6}-\left(R_{10}+R_{20}\right), \\
& R_{21} \leq J_{7}-\left(R_{10}+R_{20}\right), \\
& \left.R_{12}+R_{21} \leq J_{8}-\left(R_{10}+R_{20}\right) \mid \xi_{1}\right],
\end{aligned}
$$

where $\xi_{1}$ is the event that Case 2 happens and there is no outage at the UEs, which is defined as

$$
\begin{gathered}
\xi_{1}=\left\{g_{12}>\max \left(\sqrt{\frac{2_{2}^{\alpha R_{12}}-1}{\rho_{11}}}, g_{10}\right),\right. \\
\left.g_{21}>\max \left(\sqrt{\frac{2^{\alpha_{1} R_{21}}-1}{\rho_{22}}}, g_{20}\right)\right\} .
\end{gathered}
$$

4) Outage of the Private Parts: For the private parts, the rate constraints obtained from (9) are

$$
R_{10} \leq J_{3}, \quad R_{20} \leq J_{4}, \quad R_{10}+R_{20} \leq J_{5} .
$$

This region is similar to the classical MAC. Hence, by defining $\xi_{2}$ as the event that (17) holds, the common $\left(P_{c p}\right)$ and individual $\left(P_{1 p}, P_{2 p}\right)$ outage probabilities for private parts can be obtained as

$$
\begin{aligned}
& P_{c p}= P\left[R_{10}>J_{3}, R_{20} \leq J_{5}-J_{1} \mid \xi_{2}, \xi_{1}\right] \\
&+P\left[R_{20}>J_{4}, R_{10} \leq J_{5}-J_{2} \mid \xi_{2}, \xi_{1}\right] \\
&+P\left[R_{10} \leq J_{5}-J_{2}, R_{20}>J_{5}-J_{1},\right. \\
&\left.R_{10}+R_{20}>J_{5} \mid \xi_{2}, \xi_{1}\right], \\
& P_{1 p}= P\left[R_{10}>J_{3}, R_{20} \leq J_{5}-J_{1} \mid \xi_{2}, \xi_{1}\right] \\
&+P\left[R_{10} \leq J_{5}-J_{2}, R_{20}>J_{5}-J_{1},\right. \\
&\left.R_{10}+R_{20}>J_{5} \mid \xi_{2}, \xi_{1}\right], \\
& P_{2 p}= P\left[R_{20}>J_{4}, R_{10} \leq J_{5}-J_{2} \mid \xi_{2}, \xi_{1}\right] \\
&+P\left[R_{10} \leq J_{5}-J_{2}, R_{20}>J_{5}-J_{1},\right. \\
&\left.R_{10}+R_{20}>J_{5} \mid \xi_{2}, \xi_{1}\right] .
\end{aligned}
$$

Remark 2: Although the probabilities in (21) are in a similar form to those in [14], they are conditional probabilities that depend on the outage event for the common part in (17). Hence, the formulas in (21) cannot be evaluated in closed forms as in [14].

5) Outage at the Base Station: Since an outage for any cooperative part leads to an outage for both private information parts, the individual outage at the BS in (23) occurs with probability $\left(P_{b 1}\right)$ if the cooperative parts are in outage or the cooperative parts are decoded correctly but the private information part of $\mathrm{UE}_{1}$ is in outage. Similar analysis applies for $P_{b 2}$. The common outage occurs at the BS with probability $P_{b c}$ if the cooperative parts are in outage or the cooperative parts are decoded correctly but either or both private parts are in outage. Hence, we have

$$
\begin{aligned}
& P_{b c}=P_{c c}+\bar{P}_{c c} P_{c p}, \\
& P_{b 1}=P_{c c}+\bar{P}_{c c} P_{1 p}, \\
& P_{b 2}=P_{c c}+\bar{P}_{c c} P_{2 p},
\end{aligned}
$$

where $P_{c c}$ is given in (18), $\bar{P}_{c c}=1-P_{c c}$ and $P_{c p}, P_{1 p}$ and $P_{2 p}$ are given as in (21). 
6) Overall Outage for Case 2: The outage probability for Case 2 can now be obtained from (16) and (22). Common outage occurs if there is an outage at $\mathrm{UE}_{1}$, or there is no outage at $\mathrm{UE}_{1}$ but an outage at $\mathrm{UE}_{2}$, or there is no outage at either UE but an outage at the BS. Similar analysis holds for the individual outages. Therefore, the common $\left(P_{c 2}\right)$ and individual $\left(P_{12}, P_{22}\right)$ outage probabilities become

$$
\begin{aligned}
& P_{c 2}=P_{m 1}+\bar{P}_{m 1} P_{m 2}+\bar{P}_{m 1} \bar{P}_{m 2} P_{b c}, \\
& P_{12}=P_{m 1}+\bar{P}_{m 1} P_{m 2}+\bar{P}_{m 1} \bar{P}_{m 2} P_{b 1}, \\
& P_{22}=P_{m 1}+\bar{P}_{m 1} P_{m 2}+\bar{P}_{m 1} \bar{P}_{m 2} P_{b 2},
\end{aligned}
$$

where $\bar{P}_{m 1}=1-P_{m 1}, \bar{P}_{m 2}=1-P_{m 2}, P_{b c}, P_{b 1}$ and $P_{b 2}$ are the outage probabilities at the BS (22).

Remark 3: Since an outage at either UE will cause an outage of the common information part, and each private information part is superposed on both common parts, UE outages contribute to both the common and private outages overall.

\section{Outage Probability for Transmission Cases 3 and 4}

Case 3 occurs when $g_{12}>g_{10}, g_{21} \leq g_{20}$, which allows one way of cooperation from $\mathrm{UE}_{1}$ to $\mathrm{UE}_{2}$. The probability of this case is the same as (15) but replacing $\mu_{10}$ by $\mu_{12}$ in the numerator.

In this case, only the target rate of $\operatorname{UE}_{1}\left(R_{1}\right)$ is divided into cooperative and private target rates as $R_{1}=R_{10}+R_{12}$. The outage probability now depends on the outage probability at $\mathrm{UE}_{2}$ and the BS. Since the outage at $\mathrm{UE}_{2}$ is identical to $P_{m 2}$ given in (16), we only analyze the outage at the BS.

Similar to Case 2, the outage at the BS consists of two parts: cooperative and private outages. In this case, there is only one cooperative information part with rate constraint obtained from (9) as

$$
R_{12} \leq J_{6}-\left(R_{10}+R_{2}\right)
$$

Thus, the outage probability for the cooperative part is

$$
P_{c r}=P\left[R_{12}>J_{6}-\left(R_{10}+R_{2}\right) \mid \xi_{3}\right],
$$

where $\xi_{3}$ is the event that Case 3 happens and there is no outage at $\mathrm{UE}_{2}$, which is given as

$$
\xi_{3}=\left\{g_{12}>\max \left(\sqrt{\frac{2^{\alpha_{2} R_{12}}-1}{\rho_{11}}}, g_{10}\right), g_{21} \leq g_{10}\right\} \text {. }
$$

For the private parts, the outage probability is similar to Case 2 but with $\xi_{2}$ pertains to the event that (24) holds. Hence, the common and individual outage probabilities at the BS are given as

$$
\begin{aligned}
& P_{b c}=P_{c r}+\bar{P}_{c r} P_{c p}, \\
& P_{b 1}=P_{c r}+\bar{P}_{c r} P_{1 p}, \quad P_{b 2}=P_{c r}+\bar{P}_{c r} P_{2 p},
\end{aligned}
$$

where $P_{c p}, P_{1 p}$, and $P_{2 p}$ are given in (21) with $R_{20}=R_{2}$ and $\xi_{2}$ pertains to the event that (24) holds. Finally, the overall common $\left(P_{c 3}\right)$ and individual $\left(P_{13}, P_{23}\right)$ outage probabilities for this case are given as

$$
\begin{aligned}
& P_{c 3}=P_{m 2}+\bar{P}_{m 2} P_{b c}, \\
& P_{13}=P_{m 2}+\bar{P}_{m 2} P_{b 1}, \quad P_{23}=P_{m 2}+\bar{P}_{m 2} P_{b 2},
\end{aligned}
$$

with $P_{b c}, P_{b 1}$, and $P_{b 1}$ as in (27).

Case 4 occurs when $g_{12} \leq g_{10}, g_{21}>g_{20}$ and is simply the opposite of Case 3.

\section{Outage Rate Region}

The last two subsections provide the formulation and analysis of the outage probabilities at a given target rate pair. Some services may require target outage probabilities instead of the target rates. For these services, we can obtain the individual and common outage rate regions as follows.

Definition 1: For given target outage probabilities $\left(\beta_{1}, \beta_{2}\right)$, the individual outage rate region of the proposed D2D uplink cooperative scheme consists of all rate pairs $\left(R_{1}, R_{2}\right)$ such that

$$
P_{1}\left(R_{1}, R_{2}, \underline{\rho}\right) \leq \beta_{1}, \quad P_{2}\left(R_{1}, R_{2}, \underline{\rho}\right) \leq \beta_{2}
$$

where $\rho=\left(\rho_{10}, \rho_{20}, \rho_{11}, \rho_{22}, \rho_{13}, \rho_{23}\right)$ represents all possible power allocations satisfying the power constraints in (4). $P_{1}$ and $P_{2}$ are functions of $\left(R_{1}, R_{2}, \rho\right)$ as shown in (23) and (28). Similarly, the common outage rate region consists of all rate pairs $\left(R_{1}, R_{2}\right)$ such that $P_{c}\left(R_{1}, R_{2}, \rho\right) \leq \min \left\{\beta_{1}, \beta_{2}\right\}$ with $P_{c}$ as given in (23) and (28).

\section{Generalization to N-UE}

The previous sections consider the two-UE case as the basic model for cooperation. In practice, it can be common to have many UEs located close to each other such that cooperation among them is feasible. In this section, we generalize the two-UE scheme to $N$-UE and study the performance limit of this scheme by considering the MISO upper bound. We show that while the throughput gain diminishes as $N$ increases, the diversity improves linearly with $N$.

\section{A. Transmission Scheme and Achievable Rate Region}

The $N$-UE scheme can be described similarly to the 2-UE case. Each block is divided into $N+1$ phases with variable lengths $\alpha_{1}, \alpha_{2}, \ldots, \alpha_{N}, \alpha_{N+1}$ where $\sum_{i=1}^{N+1} \alpha_{i}=1$. Each UE splits its information $w_{k}$ into two parts $\left(w_{k k}, w_{k, N+1}\right)$. During any of the first $N$ phases, one of the UEs sends its cooperative part $w_{k k}$ while all other UEs decode it. Then, during the last phase, each UE sends all cooperative parts together with its private part $w_{k, N+1}$. Using encoding and joint decoding techniques similar to the 2-UE case, we obtain the following rate region. 
Lemma 3: An achievable rate region for N-UE consists of all N-tuples $\left(R_{1}, R_{2}, \ldots, R_{N}\right)$ satisfying

$$
\begin{aligned}
R_{\mathcal{T}} \leq & \left(\sum_{k \in \mathcal{T}} \alpha_{k} \min _{j \in[1: N], j \neq k} \log \left(1+g_{k j}^{2} \rho_{k k}\right)\right) \\
& +\alpha_{N+1} \log \left(1+\sum_{k \in \mathcal{T}} g_{k 0}^{2} \rho_{k 0}\right), \\
R_{S} \leq & \left(\sum_{k=1}^{N} \alpha_{k} \log \left(1+g_{k 0}^{2} \rho_{k k}\right)\right) \\
& +\alpha_{N+1} \log \left(1+\sum_{k=1}^{N} g_{k 0}^{2} \mathcal{P}\right. \\
& \left.+2 \sum_{i=1}^{N-1} \sum_{k=i+1}^{N} g_{i 0} g_{k 0} \sqrt{\left(\rho_{i, N+1} \rho_{k, N+1}\right)}\right)
\end{aligned}
$$

for some $\alpha_{k} \geq 0, k \in\{1: N\}, \quad \sum_{k=1}^{N} \alpha_{k} \leq 1$ and power allocation set $\left(\rho_{k 0}, \rho_{k k}, \rho_{k}\right)$ for UE $k$ satisfying $\alpha \rho_{k k}+$ $\alpha_{N+1}\left(\rho_{k 0}+\rho_{k, N+1}\right) \leq \mathcal{P}$ and all subsets $\mathcal{T} \subseteq[1: N]$. Here, $R_{\mathcal{T}}=\sum_{k \in \mathcal{T}} R_{k}$ and $R_{S}=\sum_{i=1}^{N} R_{i}$ is the total sum rate.

Proof: Similar to the proof of Theorem 1.

\section{B. MISO Upper Bound}

To study the performance limit as the number of cooperating UEs increases, we consider the MISO case as an upper bound for proposed scheme as it simplifies the analysis and gives a good upper bound on the actual performance of the proposed scheme. This case can also occur in practice in a situation where the group of $N$ UEs is in close proximity such that all cooperative links among the UEs are much stronger than the direct links to the BS. In this case, $R_{\mathcal{T}}$ in (30) will be redundant since all $g_{k j} \gg g_{k 0}$. Then, the only effective rate constraint is the sum rate. Moreover, $\alpha_{i} \rightarrow 0$ for $i \in\{1: N\}$ and $\alpha_{N+1} \rightarrow 1$ since UEs can exchange their information in very short times and spend most of the time in coherent transmission [19].

1) Achievable Rate: Based on the above discussion, the achievable (sum) rate $\left(R_{S}=R_{1}+R_{2}+\ldots+R_{N}\right)$ in (30) is the dominant constraint and is expressed as

$$
R_{S} \leq \log \left(1+\sum_{k=1}^{N} g_{k 0}^{2} \mathcal{P}+2 \sum_{i=1}^{N-1} \sum_{k=i+1}^{N} g_{i 0} g_{k 0} \mathcal{P}\right)=J_{S}
$$

Consider the case where all the UEs are closely located as an upper bound on performance, we obtain the following lemma.

Lemma 4: The percentage of the rate gain approaches 0 as $N \rightarrow \infty$.

Proof: This is straightforward by taking the sum rate and analyzing the gain with increasing $N$.

Although the rate gain is diminishing with $N$, simulation results shown later in Section VII-D suggest that the gain is still worthwhile for up to 3 UEs cooperating, where a throughput gain of $20 \%$ or more is still feasible. For two UEs cooperating, the throughput gain can be up to $80 \%$.

2) Outage Probability: Considering outage performance in fading channel, the impact is the opposite. Although some cooperative links can be weaker than the direct links for specific channel realizations even when the UEs are much closer to each other than to the BS, the probability of having these cases is marginal. Hence, the sum rate constraint is still the dominant one that determines the outage performance. Then, provided that each UE has an independent fading channel to the BS, the outage probability for a specific target sum rate $R_{S}^{t}$ can be approximated as $\bar{P}_{o} \approx P\left[R_{s}^{t}>J_{S}\right]$ where $J_{S}$ is given in (31). This outage probability reduces significantly with increasing $N$ such that the diversity order is linear in $N$. Section VII-D illustrates some numerical results for the $N$-UE throughput and outage performances.

\section{Comparison With Frequency Division Schemes}

In this section, we compare the proposed TDD scheme with the existing half-duplex schemes based on FDD or CDMA in [9], [10], and [12]. We show that the proposed scheme achieves the same or better rate region while has simpler transmit signals and significantly shorter decoding delay. Moreover, we formulate the outage probability for the existing schemes as they are unavailable in these prior works.

\section{A. Three-Band Frequency Division}

Based on the original information-theoretic scheme in [9], [11], FDD can be used in the proposed scheme instead of TDD. In FDD implementation, the bandwidth of each transmission block is divided into 3 bands and the transmissions in the first 2 bands are similar to the first 2 phases in the TDD scheme except that both UEs transmit at the same time (on different frequency bands). In the 3rd band, both users will transmit concurrently. However, because in the same block of time, the two users are still exchanging current cooperative information on the first 2 bands, then in the 3rd band, they can only send the previous and not the current cooperative information as in [9] and [11]. Therefore, FDD implementation requires block Markov signaling structure which requires backward decoding with long block delay, or sliding window decoding with one block delay. In [10], a half-duplex cooperative OFDDMA system with $\mathrm{N}$ subchannels is proposed where these subchannels are divided into 3 sets. Considering these 3 sets as the 3 phases of the FDD scheme, the transmission and the achievable rate regions in these two schemes are similar.

In comparison, for 3-band FDD and 3-set OFDDMA, the information dependency between consecutive blocks complicates the signaling by requiring a block Markov signal structure. The proposed scheme, by using TDD, overcomes this block Markov requirement and allows the forwarding of information in the same block. Moreover, backward or sliding window decoding is required for FDD implementation because of the block Markov structure, which for Gaussian channel leads to the same achievable rate region of the proposed scheme but with at least one block delay whereas the proposed scheme incurs no block decoding delay. Based on this discussion, we obtain the following corollary.

Corollary 3: The proposed 3-phase TDD scheme achieves the same rate region of the 3-band FDD or the 3-set OFDDMA scheme while having simpler transmit signals and shorter decoding delay. 


\section{B. Two-Band Frequency Division}

In [12], another half-duplex scheme is proposed based on FDD. In each block, the bandwidth is divided into two bands with widths $\beta$ and $\bar{\beta}=1-\beta$. Each band is divided by half into two sub-bands. In the first band, $\mathrm{UE}_{2}$ works as a relay for $\mathrm{UE}_{1}$ while the opposite happens in the second band. In the first sub-band, $\mathrm{UE}_{1}$ sends its information with $\rho_{12}$ power and $\mathrm{UE}_{2}$ decodes it. In the second sub-band, $\mathrm{UE}_{1}$ and $\mathrm{UE}_{2}$ allocate the powers $\rho_{1}^{(1)}$ and $\rho_{1}^{(2)}$, respectively to send the previous information of $\mathrm{UE}_{1}$ to the BS. The opposite happens in the second band. The BS employs sliding window decoding. The achievable rate of this scheme consists of the rate pairs $\left(R_{1}, R_{2}\right)$ satisfying [12]

$$
\begin{aligned}
& R_{1} \leq \min \left\{A_{1}, A_{3}\right\}, R_{2} \leq \min \left\{A_{2}, A_{4}\right\}, \\
& A_{1}=0.5 \beta \log \left(1+g_{12}^{2} \rho_{12}\right), A_{2}=0.5 \bar{\beta} \log \left(1+g_{21}^{2} \rho_{21}\right), \\
& A_{3}=0.5 \beta \log \left(1+g_{10}^{2} \rho_{12}+\left(g_{10} \sqrt{\rho_{1}^{(1)}}+g_{20} \sqrt{\rho_{1}^{(2)}}\right)^{2}\right), \\
& A_{4}=0.5 \bar{\beta} \log \left(1+g_{20}^{2} \rho_{21}+\left(g_{10} \sqrt{\rho_{2}^{(1)}}+g_{20} \sqrt{\rho_{2}^{(2)}}\right)^{2}\right),
\end{aligned}
$$

for some $0 \leq \beta \leq 1$ and power allocation satisfying

$$
\begin{aligned}
& \beta\left(\rho_{12}+\rho_{1}^{(1)}\right)+\bar{\beta} \rho_{2}^{(1)} \leq \mathcal{P}_{1}, \\
& \bar{\beta}\left(\rho_{21}+\rho_{2}^{(2)}\right)+\beta \rho_{1}^{(2)} \leq \mathcal{P}_{2} .
\end{aligned}
$$

Corollary 4: The 2-band scheme has longer delay and smaller rate region than the proposed scheme.

Proof: The 2-band scheme has one block delay because of using sliding window decoding. Moreover, the scheme uses neither information splitting nor superposition coding. These two techniques, which are employed in the proposed scheme, enlarge the rate region as shown in Appendix A.

\section{Outage Probability Analysis}

Next, we derive the outage probability for the existing schemes in [9], [10], [12] as outage results are unavailable in these previous works.

1) Outage for the 3-Band Frequency-Division Transmission: For the 3-band FDD scheme derived from [9] and the OFDDMA scheme in [10], the outage probability is given as follows.

Corollary 5: The outage probability for the 3-band FDD or OFDDMA scheme is similar to the proposed TDD scheme except that the cooperative common outage for Case 2 in (18) is replaced with

$$
P_{c c}=1-P\left[R_{12}+R_{21} \leq J_{8}-\left(R_{10}+R_{20}\right) \mid \xi_{1}\right]
$$

Proof: Since the BS in both schemes employs backward decoding, the rate constraints at the BS are similar to (9) but without $R_{1}+R_{20} \leq J_{6}$ and $R_{10}+R_{2} \leq J_{7}$. Hence, these 2 constraints are removed from the cooperative common outage in (34).

2) Outage for the 2-Band Frequency-Division Transmission: For this scheme, the outage probability can be formulated from the achievable rate region in (32) following similar procedure for the outage of the proposed scheme, where in

- Case 1, direct transmission is used with $\rho_{1}^{(2)}=\rho_{2}^{(1)}=0$.

- Case 2, cooperation from both UEs.

- Case 3, cooperation from $\mathrm{UE}_{1}$ and direct transmission form $\mathrm{UE}_{2}$ with $\rho_{2}^{(1)}=0$.

- Case 4 , cooperation from $\mathrm{UE}_{2}$ and direct transmission form $\mathrm{UE}_{1}$ with $\rho_{1}^{(2)}=0$.

Then, the outage probability is given as follows.

Corollary 6: For the 2-band FDD scheme with the achievable rate region in (32), the common outage probability is given as in (14) but with

$$
\begin{aligned}
P_{c 1}= & P\left[R_{1}>A_{3}, R_{3} \leq A_{4}\right]+P\left[R_{1}>A_{3}, R_{3}>A_{4}\right] \\
& +P\left[R_{1} \leq A_{3}, R_{3}>A_{4}\right] \\
P_{c 2}= & P_{m 1}+\bar{P}_{m 1} P_{m 2}+\bar{P}_{m 1} \bar{P}_{m 2} P_{c 1} \\
P_{c 3}= & P_{m 1}+\bar{P}_{m 1} P_{c 1}, P_{c 4}=P_{m 2}+\bar{P}_{m 2} P_{c 1} .
\end{aligned}
$$

The individual outage probabilities are formulated similarly. However, since in each bands, one UE works as a relay for the other UE, the outage at one UE will lead to an outage of the other UE information at the BS and not both information as in the proposed scheme. Hence, for $\mathrm{UE}_{1}$ outage, we have

$$
\begin{aligned}
& \left.P_{11}=P\left[R_{1}>A_{3}, R_{3} \leq A_{4}\right]\right]+P\left[R_{1}>A_{3}, R_{3}>A_{4}\right], \\
& P_{12}=P_{m 2}+\bar{P}_{m 2} P_{11}, P_{13}=P_{12}, P_{14}=P_{11} .
\end{aligned}
$$

The outage at $\mathrm{UE}_{2}$ is formulated similarly.

Proof: Obtained following similar procedure of the proposed scheme.

\section{Tradeoff Between Decoding Delay and Rate Constraints}

Comparison between the proposed TDD scheme and the 3-band FDD and OFDDMA schemes in [10] reveals the following interesting trade-offs among decoding delay, rate constraints and outage performance. Based on formula (9) and the proof of Corollary 5, the BS can decode with fewer rate constraints if it is allowed longer decoding delay, as it can use more received signals in order to have better estimation of the transmitted information. Specifically, the OFMA scheme in [10] employs backward decoding where the BS, at each block, decodes the current private information and the previous cooperative information given that it knows the current cooperative information. This knowledge reduces the error events and hence, relaxes the rate constraints stemmed from the decoding at the BS. Therefore rate constraints $J_{6}$ and $J_{7}$ are not present in the FDD backward decoding implementation but are present in our proposed TDD scheme.

For application in wireless channels, however, this difference in decoding rate constraints does not matter for the overall achievable rate region, as shown in Corollary 1 and Corollary 3. This equivalency happens since after we combine the rate constraints from the UEs and the BS, we then find as in Corollary 1 that the additional constraints, stemmed from the decoding at the BS, are redundant. For outage performance, however, all decoding rate constraints matter as we need to separately consider the outages at UEs and the BS. The difference in decoding rate constraints then leads to different outage formulas as shown in (18) and (34). Nevertheless, numerical results in Section VII 


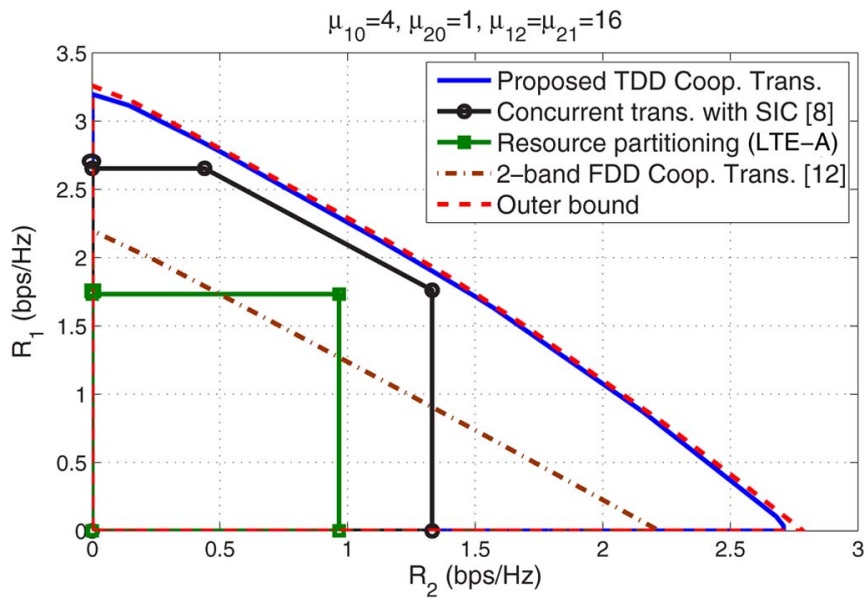

Fig. 5. Achievable rate regions and outer bounds for asymmetric half-duplex cooperative transmissions.

show that both the 3-band FDD scheme and our 3-phase TDD scheme have the same outage performance, which suggests that the additional rate constraints in our TDD scheme do not matter in outage performance either.

\section{NuMERICAL RESUlTS}

In this section, we provide numerical results and analysis for the achievable rate region, outer bound, outage probabilities and outage rate region derived in Sections III-VI. In these simulations, all the links are Rayleigh fading channels with parameters specified in each simulation. All the simulations are obtained using $10^{5}$ samples for each fading channel unless otherwise stated. Fig. 5 shows the ergodic achievable rate regions, Figs. 6-8 show the outage performance and Figs. 9 and 10 show performance gain for the MISO upper bound of the $N$-UE scheme.

\section{A. Achievable Rate Region}

Fig. 5 is obtained with $\mathcal{P}_{1}=\mathcal{P}_{2}=2$ with all possible power allocations and phase durations. It compares between the achievable rate region of the proposed and existing transmissions and the outer bound for asymmetric channels. Results are plotted with $\mu_{10}=4$ and $\mu_{20}=1$ while $\mu_{12}=\mu_{21}=16$.

As discussed in Section III-E, results show that the achievable rate region of the proposed TDD cooperative transmission is close to the outer bound since the ratios $\mu_{12} / \mu_{10}$ and $\mu_{21} / \mu_{20}$ are high. Moreover, $\mathrm{UE}_{2}$ that has weaker link to the BS obtains higher gain in the individual rate than $\mathrm{UE}_{1}$. This is because $\mathrm{UE}_{1}$ has stronger link to the $\mathrm{BS}$ and can work as a relay for $\mathrm{UE}_{2}$ information. Comparing with other transmissions, the 3-band FDD scheme in [9], [10] achieves the same rate region of the proposed scheme as mentioned in Corollary 5. However, the proposed 3-phase TDD cooperative transmission outperforms the non-cooperative scheme including the resource partitioning with orthogonal transmission (LTE-A) and the concurrent transmission with SIC where both UEs transmit directly to the destination during the entire resource block without cooperation. The proposed scheme also outperforms the 2-band FDD scheme in [12] since neither rate splitting nor

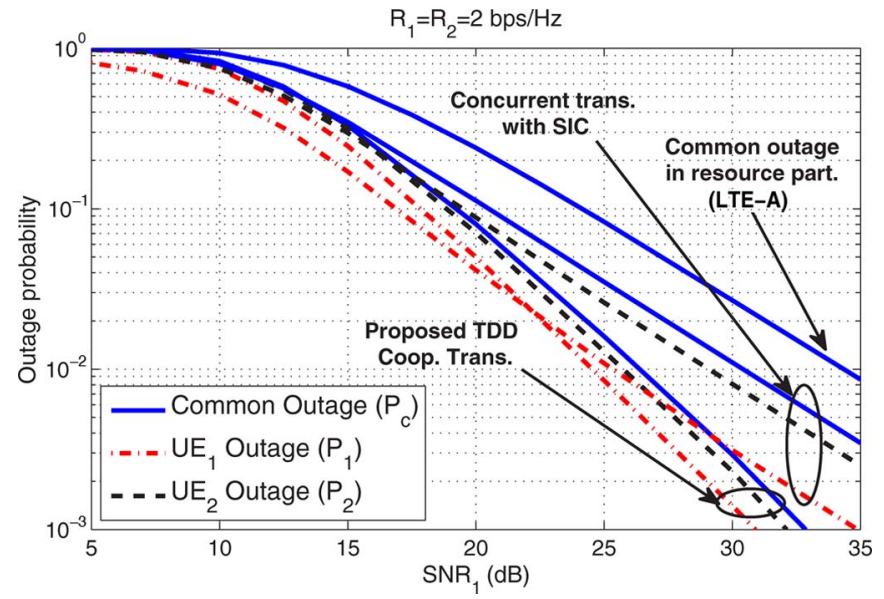

Fig. 6. Comparison between the proposed scheme and other uplink strategies in terms of common and individual outage probabilities vs $\mathrm{SNR}_{1}$.

superposition coding is employed in 2-band FDD scheme as shown in Corollary 4.

\section{B. Outage Probability}

We now provide numerical results for the formulated outage probabilities and outage rate region. The simulation settings and channel configuration are: $d_{10}=20, d_{20}=30, d_{12}=d_{21}=12$, $\mathcal{P}_{1}=\mathcal{P}_{2}=\rho$ and $\gamma=2.4$. The average channel gain for each link is given as $\mu_{i j}=\frac{1}{d i j^{\gamma}}$. With these settings, we define the average received $\mathrm{SNR}$ at the $\mathrm{BS}$ for signals from $\mathrm{UE}_{1}\left(\mathrm{SNR}_{1}\right)$ and $\mathrm{UE}_{2}\left(\mathrm{SNR}_{2}\right)$ as follows.

$$
\begin{aligned}
& \mathrm{SNR}_{1}=10 \log \left(\frac{\mu_{10} \rho}{d_{10}^{\gamma}}\right), \\
& \mathrm{SNR}_{2}=10 \log \left(\frac{\mu_{20} \rho}{d_{20}^{\gamma}}\right)=\mathrm{SNR}_{1}+10 \log \left(\frac{\mu_{20} d_{10}^{\gamma}}{\mu_{10} d_{20}^{\gamma}}\right) .
\end{aligned}
$$

The phase durations are set as follows: case 2: $\alpha_{1}=\alpha_{2}=0.25$ and $\alpha_{3}=0.5$, case 3: $\alpha_{1}=0.4$ and $\alpha_{3}=0.6$ and case $4: \alpha_{2}=$ 0.4 and $\alpha_{3}=0.6$. We fix the phase duration to simplify computation while the optimal power allocations and rate splitting are obtained numerically. For the 2-band FDD scheme in (32) [12], we fix the 2 bandwidths to half $(\beta=\bar{\beta}=0.5)$ for all cases.

Fig. 6 shows the outage probabilities versus $\mathrm{SNR}_{1}$ for the proposed scheme and concurrent transmission with SIC. Results confirm our expectation that the common outage probability is higher than individual outage probabilities. Moreover, at equal transmission rates, the individual outage probability for $\mathrm{UE}_{2}$ is higher than $\mathrm{UE}_{1}$ since $\mathrm{UE}_{2}$ has weaker direct link. For low SNR, the non-cooperative concurrent transmission with SIC scheme has lower outage probability than cooperative scheme, but the outage in this range is too high for practical interest (above 10\%). As SNR increases, the cooperative scheme starts outperforming the non-cooperative scheme. This happens because in the cooperative scheme, each UE transmits over a fraction of time instead of the whole time as in the concurrent transmission with SIC. Moreover, both UEs use part of their power to exchange information such that they transmit coherently in the 3rd phase. At low SNR, the coherent transmission has lower effect compared with the power loss in exchanging information; hence, the concurrent transmission with SIC 


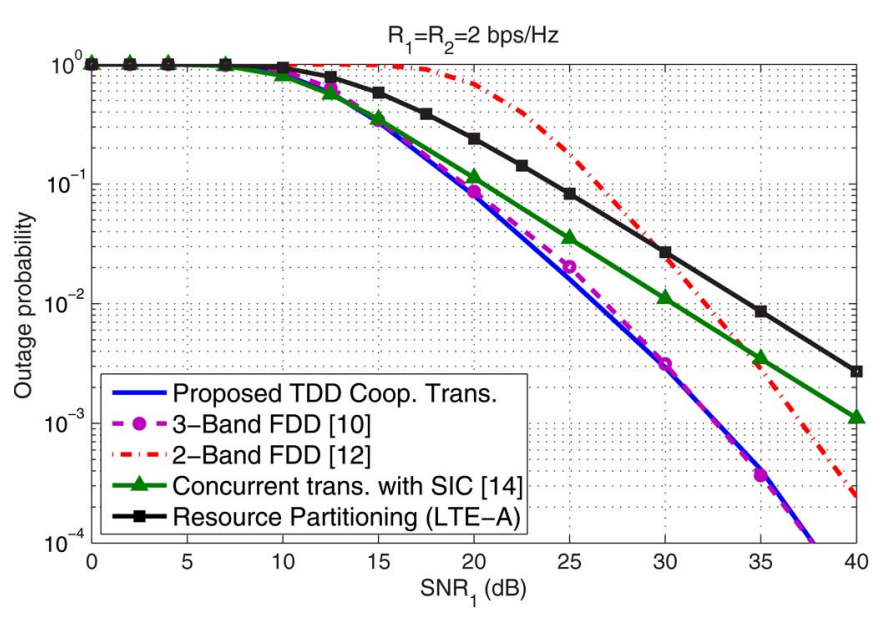

Fig. 7. Comparison between the proposed and existing cooperative schemes in terms of common outage probabilities vs $\mathrm{SNR}_{1}$.

outperforms the cooperative scheme. As SNR increases, however, the gain obtained from coherent transmission becomes dominant such that the cooperative scheme outperforms the concurrent transmission with SIC. These results are obtained with arbitrary fixed phase durations; thus if they are optimally chosen, the cooperative scheme will outperform the non-cooperative scheme at an even lower SNR. Fig. 6 also shows that the diversity order of the cooperative scheme is 2 . This result is in contrary to that in [17] which shows that decode-forward scheme for half-duplex relay channel achieves a diversity order of 1 only. The difference comes from the fact that in [17], the source only transmits in the 1st phase and there is no coherent transmission in the 2 nd phase while the relay always decodes even when its link with the source is weak. However, in our scheme, there is a coherent transmission in 2nd phase and each UE only decodes if the cooperative link is stronger than the direct link. Intuitively, our scheme always requires 2 links to be weak in order to lose the information of any UE [17]. Consider the information of $\mathrm{UE}_{1}$, if the cooperative link is weak, this information will be lost if the direct link of $\mathrm{UE}_{1}$ is also weak. If the cooperative link is strong, this information will be lost if both direct links are weak.

Fig. 7 compares the common outage probabilities of the proposed transmission, 3-band FDD [10], 2-band FDD [12], concurrent with SIC [8] and resource partitioning with orthogonal transmission (current LTE-A). Results show that compared with non-cooperative transmissions, cooperation improves the diversity whether using the proposed TDD transmission, 3 or 2-band FDD. However, 2-band FDD transmission requires more power to start outperforming the non-cooperative transmissions. The proposed TDD scheme and 3-band FDD transmissions have the same outage performance although their outage formulas are slightly different (Corollary 3 ).

\section{Outage Rate Region}

Fig. 8 shows the common and individual outage rate regions when the target outage probability for both UEs is $1 \%$. While the target outages are the same, the rate regions are asymmetric because the direct links are different. Results show that the proposed cooperative transmission has larger regions than

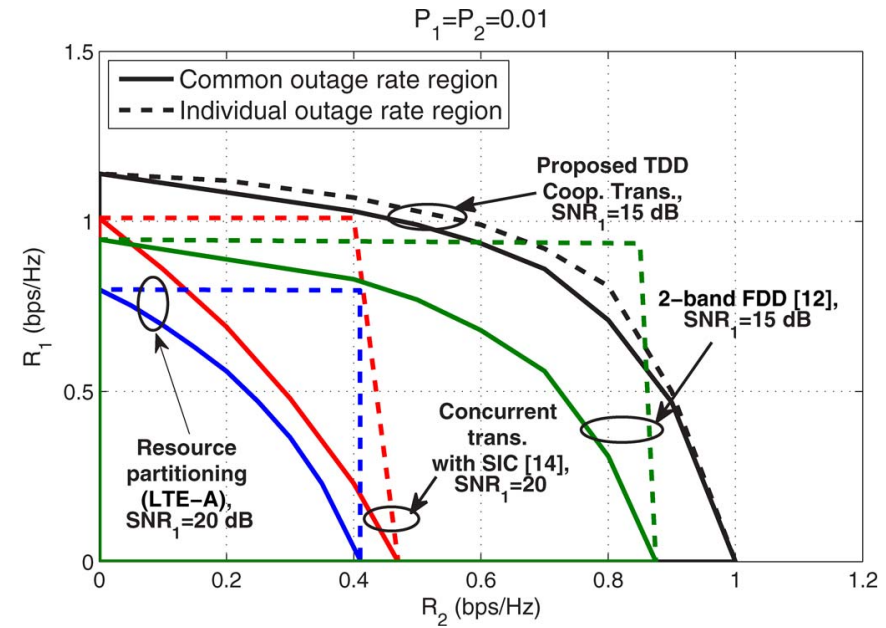

Fig. 8. Common and individual outage rate regions for the proposed and existing schemes at $P_{1}=P_{2}=1 \%$ outage and different $\mathrm{SNR}_{1}$.

the non-cooperative ones (resource partitioning or concurrent transmission with SIC) even when it has a lower transmit power lower by $5 \mathrm{~dB}$. Note that considering the outage performance in Fig. 6, the gap between the cooperative and non-cooperative transmissions will increase if the target outage probability decreases, and vice versa.

The 3-band $\mathrm{UE}_{1}$ [9], [10] has the same performance as the proposed TDD transmission. For the 2-band FDD transmission [12], while the common outage region is always included in that of the proposed transmission, the individual outage region unexpectedly intersects with that of the proposed transmission. This intersection may occur since we fix the phases of the proposed transmissions and the bandwidths for 2-band transmission in [12]. While fixing them simplifies the computations, these selections can be suboptimal and lead to unexpected results. The largest regions should be obtained from all possible phase durations and bandwidths.

\section{N-UE Case}

Results in Figs. 9 and 10 are obtained with $10^{6}$ samples for each fading channel. Fig. 9 shows the percentage of rate gain versus the number of UEs $(N)$. Results clearly show that the throughput is significant when using 2 UEs. By increasing $N$, the throughput keeps increasing but with lower percentage till the gain is less than $10 \%$ when $N>5$.

Fig. 10 illustrates the outage probabilities for different $N$. Results show that the diversity order increases linearly with $\mathrm{N}$, showing a significant improvement in reliability with increasing $N$. We note that results in both Figs. 9 and 10 are upper bounds on the actual $N$-UE cooperative performance.

\section{CONCLUSION}

We have analyzed both the instantaneous achievable rate region and the outage probability of a time-division hybrid D2D-infrastructure cooperative scheme in uplink cellular communication. The scheme employs rate splitting, superposition coding, partial decode-forward relaying and ML decoding in a 3-phase half-duplex transmission. 


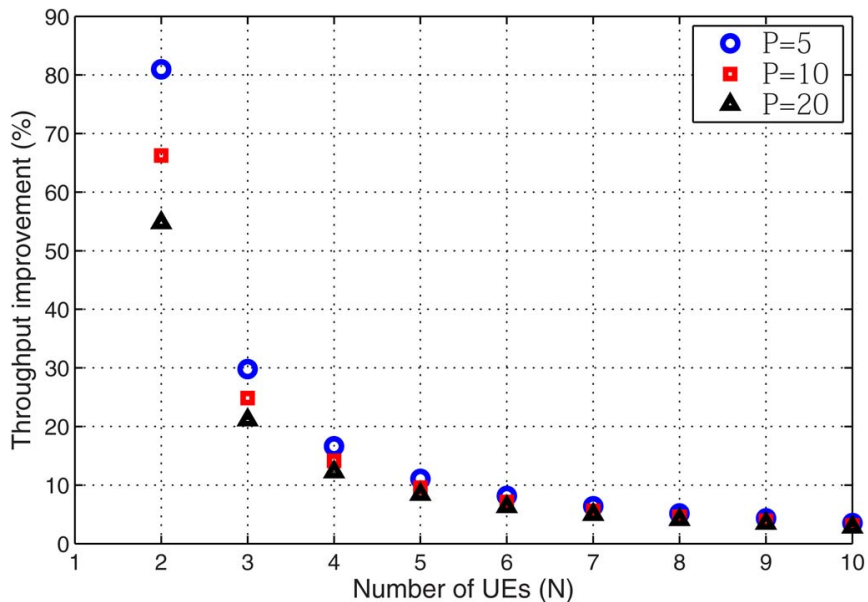

Fig. 9. Percentage of rate gain vs the number of UEs $(N)$ with $\mu_{i 0}=1$ for all $i \in\{1: N\}$ and different values of $\mathcal{P}$.

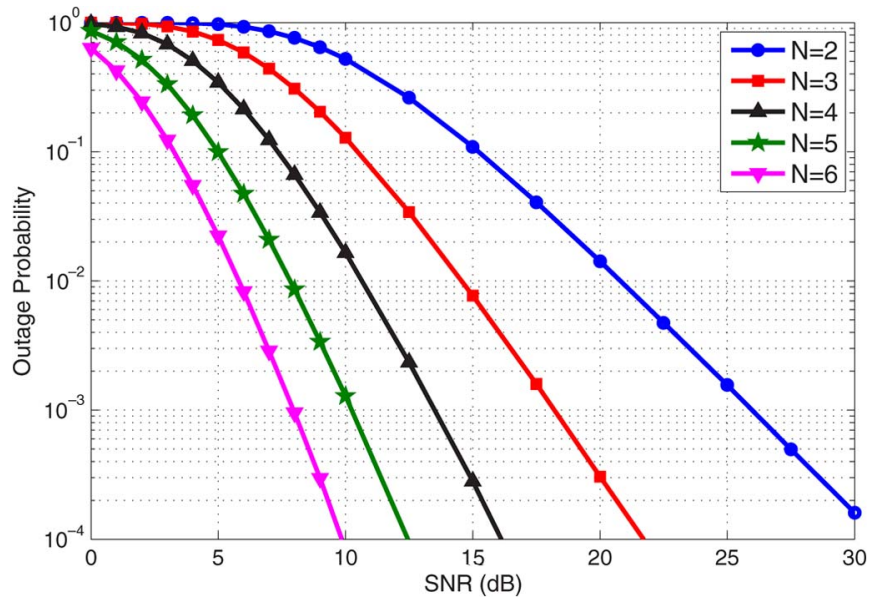

Fig. 10. Outage probabilities with different values of $N, R_{S}^{t}=5 \mathrm{bps} / \mathrm{Hz}$ and $\mu_{i 0}=1$ for all $i \in\{1: N\}$.

The TDD implementation is simpler than existing FDD schemes while achieving comparable or better rate performance. When applied to fading channels, outage probabilities can be computed based on outages at the UEs and BS. We formulate for the first time both the common and individual outage probabilities for a cooperative transmission scheme and also analyze outage of existing FDD schemes. Numerical results show significant gains in both achievable rates and outage performance by the proposed TDD cooperative scheme. These results suggest the use of cooperation among a small number of UEs for most practical ranges of SNR, except very low SNR where non-cooperative schemes may have better outage performance. For future work, it is of interest to apply of the proposed scheme in a multi-cell multi-tier system and analyze its impact on the entire network performance.

\section{APPENDIX A}

Proof of the Achievable Region in Theorem 1

First, the transmission rates are related to the size of the information sets $(\mathcal{I}, \mathcal{J}, \mathcal{K}$, and $\mathcal{L})$ as

$$
R_{12}=\frac{1}{n} \log |\mathcal{I}|, \quad R_{10}=\frac{1}{n} \log |\mathcal{J}|,
$$

$$
R_{21}=\frac{1}{n} \log |\mathcal{K}|, \quad R_{20}=\frac{1}{n} \log |\mathcal{L}| .
$$

The error events at UEs can be analyzed as in [20], [25]. To make these error probabilities approach zero, $R_{12}$ and $R_{21}$ must satisfy the first two constraints involving $I_{1}$ and $I_{2}$ in (6).

For the decoding at the BS, the maximum rate $J_{\star}$ achievable for the given channel realization in (9) is obtained by upper bounding error events resulted from the decoding rule in (8). Assuming all messages are equally likely, the error probability does not depend on which tuple $(i, k, j, l)$ was sent. Without loss of generality, assume that $\{i=k=j=k=1\}$ was sent. Then, $J_{\star}$ ensures that the probability of error event $E_{\star}$ approaches zero as the transmit sequence lengths increase, where for

- $J_{3}: E_{3}=\{\hat{i}=\hat{k}=\hat{l}=1, \hat{j} \neq 1\}$, only the private part of $\mathrm{UE}_{1}$ is decoded incorrectly.

- $J_{4}: E_{4}=\{\hat{i}=\hat{k}=\hat{j}=1, \hat{l} \neq 1\}$, only the private part of $\mathrm{UE}_{2}$ is decoded incorrectly.

- $J_{5}: E_{5}=\{\hat{i}=\hat{k}=1,(\hat{j}, \hat{l}) \neq 1\}$, both private parts are decoded incorrectly.

- $J_{6}: E_{6}=\{\hat{k}=1,(\hat{i}, \hat{j}, \hat{l}) \neq 1\}$, both private parts of two UEs and the cooperative part of $\mathrm{UE}_{1}$ are decoded incorrectly.

- $J_{7}: E_{7}=\{\hat{i}=1,(\hat{k}, \hat{j}, \hat{l}) \neq 1\}$, similar to $J_{6}$ but with cooperative part of $\mathrm{UE}_{2}$ decoded incorrectly.

- $J_{8}: E_{8}=\{(\hat{i}, \hat{k}, \hat{j}, \hat{l}) \neq 1\}$, all information parts are decoded incorrectly.

To analyze the upper bounds for the probabilities of these error events, we will first divide them into 2 groups where error probabilities in each group have similar analysis. The 1st group contains: $\left(P_{E_{3}}, P_{E_{4}}, P_{E_{5}}\right)$ and the 2nd group contains: $\left(P_{E_{6}}, P_{E_{7}}, P_{E_{8}}\right)$ Since the error analysis for the second group is more complicated, we only analyze the 6th error event $\left(E_{6}\right)$. The error analysis for the first group can be obtained similarly.

Define $\vartheta_{2}$ as the event that (38) holds.

$$
\begin{aligned}
& P\left(y \mid x_{1}(j, i, 1), x_{2}(l, i, 1)\right) \geq P\left(y \mid \tilde{x}_{1}, \tilde{x}_{2}\right) \\
& \leftrightarrow P\left(y_{1} \mid x_{11, i}\right) P\left(y_{2} \mid x_{22,1}\right) P\left(y_{3} \mid x_{13,(j, i, 1)}, x_{23,(l, i, 1)}\right) \\
& \geq P\left(y_{1} \mid \tilde{x}_{11}\right) P\left(y_{2} \mid \tilde{x}_{22}\right) P\left(y_{3} \mid \tilde{x}_{13}, \tilde{x}_{23}\right) \\
& \leftrightarrow P\left(y_{1} \mid x_{11, m_{12}}\right) P\left(y_{3} \mid x_{13,(j, i, 1)}, x_{20,(l, i, 1)}\right) \\
& \geq P\left(y_{1} \mid \tilde{x}_{11}\right) P\left(y_{3} \mid \tilde{x}_{13}, \tilde{x}_{23}\right) .
\end{aligned}
$$

Then, the probability of this event is

$$
\begin{aligned}
P\left(\vartheta_{2}\right)= & \sum_{x_{11}, u_{3}, x_{13}, x_{23}} P\left(x_{11, i}\right) p\left(u_{3,(i, 1)}\right) \\
& \times P\left(x_{13,(j, i, 1)} \mid u_{3,(i, 1)}\right) P\left(x_{23,(l, i, 1)} \mid u_{3,(i, 1)}\right) .
\end{aligned}
$$

This probability can be bounded as follows [20], [25].

$$
\begin{gathered}
P\left(\vartheta_{2}\right) \leq \sum_{x_{11}, u_{3}, x_{13}, x_{23}} P\left(x_{11, i}\right) P\left(x_{13,(j, i, 1)}\right) P\left(x_{23,(l, i, 1)}\right) \\
\quad \times\left(\frac{P\left(y_{1} \mid x_{11, i}\right)}{P\left(y_{1} \mid x_{11}(1)\right)}\right)^{s}\left(\frac{P\left(y_{3} \mid x_{13,(j, i, 1)}, x_{23,(l, i, 1)}\right)}{P\left(y_{3} \mid x_{13}(1,1,1), x_{23}(1,1,1)\right)}\right)^{s}
\end{gathered}
$$


for any $s>0$. Now, let $\vartheta$ be the event that (38) holds for some $m_{12} \neq 1$ and any $j$, and $l$. Then for any $0 \leq \rho \leq 1$, the probability of the event $\vartheta$ can be expressed as follows [20], [25].

$$
\begin{aligned}
P(\vartheta) \leq & \left(\sum_{i, j, l} P\left(\vartheta_{2}\right)\right)^{\rho}=(|\mathcal{I}|-1)^{\rho}|\mathcal{J}|^{\rho}|\mathcal{L}|^{\rho} \\
& \times\left[\sum_{x_{11}, u_{3}, x_{13}, x_{23}} P\left(x_{11}\right) P\left(x_{13}\right) P\left(x_{23}\right)\right. \\
& \left.\times\left(\frac{P\left(y_{1} \mid x_{10}\right)}{P\left(y_{1} \mid \tilde{x}_{10}\right)}\right)^{s}\left(\frac{P\left(y_{3} \mid x_{13}, x_{23}\right)}{P\left(y_{3} \mid \tilde{x}_{13}, \tilde{x}_{23}\right)}\right)^{s}\right]^{\rho}
\end{aligned}
$$

where $\boldsymbol{i} \in\{2, \ldots,|\mathcal{I}|\}, \boldsymbol{j} \in\{1, \ldots,|\mathcal{J}|\}$ and $\boldsymbol{l} \in\{1, \ldots,|\mathcal{L}|\}$. Then, the probability of interest, $P_{E_{6}}$, has an upper bound:

$$
\begin{aligned}
P_{E_{6}} \leq \sum_{y_{13}, x_{11}, x_{13}, x_{23}} P\left(y_{1} \mid \tilde{x}_{11}\right) & P\left(y_{3} \mid \tilde{x}_{13}, \tilde{x}_{23}\right) \\
\times & P\left(\tilde{x}_{11}\right) P\left(\tilde{x}_{13}\right) P\left(\tilde{x}_{23}\right) P(\vartheta) .
\end{aligned}
$$

By combining the last two equations and by choosing $s=$ $1 /(1+\rho), P_{E_{6}}$ can be written as

$$
\begin{aligned}
P_{E_{6}} \leq(|\mathcal{I}|-1)^{\rho}|\mathcal{J}|^{\rho}|\mathcal{L}|^{\rho} \sum_{y_{13}}\left[\sum_{x_{11}} P\left(\tilde{x}_{11}\right)\left(P\left(y_{1} \mid \tilde{x}_{11}\right)\right)^{\frac{1}{1+\rho}}\right]^{1+\rho} \\
\times\left[\sum_{x_{13}, x_{23}} P\left(\tilde{x}_{13}, \tilde{x}_{23}\right)\left(P\left(y_{3} \mid \tilde{x}_{13}, \tilde{x}_{23}\right)\right)^{\frac{1}{1+\rho}}\right]^{1+\rho} .
\end{aligned}
$$

Since the channel is memoryless, $P_{E_{6}}$ can be expanded as follows.

$$
\begin{aligned}
& P_{E_{6}} \leq(|\mathcal{I}|-1)^{\rho}|\mathcal{J}|^{\rho}|\mathcal{L}|^{\rho} \\
& \times \sum_{y_{1,1}^{\alpha_{1} n}, y_{3, \alpha_{d}}^{n}}\left[\sum_{x_{11,1}^{\alpha_{1}} n} \prod_{t=1}^{\alpha_{1} n} P\left(x_{11_{t}}\right)\left(P\left(y_{1_{t}} \mid x_{11_{t}}\right)\right)^{\frac{1}{1+\rho}}\right]^{1+\rho} \\
& {\left[\sum_{x_{13, \alpha_{d}}^{n}, x_{23, \alpha_{d}}^{n}} \prod_{i=\alpha_{d}}^{n} P\left(x_{13_{t}}, x_{23_{t}}\right)\left(P\left(y_{3_{t}} \mid x_{13_{t}}, x_{23_{t}}\right)\right)^{\frac{1}{1+\rho}}\right]^{1+\rho} .}
\end{aligned}
$$

Then, by interchanging the order of the products and the summations, (40) can be simplified to:

$$
\begin{aligned}
& P_{E_{6}} \leq(|\mathcal{I}|-1)^{\rho}|\mathcal{J}|^{\rho}|\mathcal{L}|^{\rho} \\
& \times \prod_{t=1}^{\alpha_{1} n} \sum_{y_{1_{t}}}\left[\sum_{x_{11_{t}}} P\left(x_{11_{t}}\right)\left(P\left(y_{1_{t}} \mid x_{11_{t}}\right)\right)^{\frac{1}{1+\rho}}\right]^{1+\rho} \\
& \times \prod_{t=\alpha_{d}}^{n} \sum_{y_{3_{t}}}\left[\sum_{x_{13_{t}}, x_{23_{t}}} P\left(x_{13_{t}}, x_{23_{t}}\right)\left(P\left(y_{3_{t}} \mid x_{13_{t}}, x_{23_{t}}\right)\right)^{\frac{1}{1+\rho}}\right]^{1+\rho} .
\end{aligned}
$$

Since the summations are taken over the inputs and the outputs alphabets, $P_{E_{6}}$ can be expressed as

$$
\begin{gathered}
P_{E_{6}} \leq(|\mathcal{I}|-1)^{\rho}|\mathcal{J}|^{\rho}|\mathcal{L}|^{\rho} \\
\times\left(\sum_{y_{1_{t}}}\left[\sum_{x_{11_{t}}} P\left(x_{11_{t}}\right)\left(P\left(y_{1_{t}} \mid x_{11_{t}}\right)\right)^{\frac{1}{1+\rho}}\right]^{1+\rho}\right)^{\alpha_{1} n} \\
\times\left(\sum _ { y _ { 3 _ { t } } } \left[\sum_{x_{13_{t}}, x_{23_{t}}} P\left(x_{13_{t}}, x_{23_{t}}\right)\right.\right. \\
\left.\left.\times\left(P\left(y_{3_{t}} \mid x_{13_{t}}, x_{23_{t}}\right)\right)^{\frac{1}{1+\rho}}\right]^{1+\rho}\right)^{\alpha_{3} n} .
\end{gathered}
$$

Following [25], $(\mathcal{I}-1) \mathcal{J} \mathcal{L}$, has the following upper bound: $(|\mathcal{I}|-1)|\mathcal{J}||\mathcal{L}|<2^{n\left(R_{12}+R_{10}+R_{20}+\frac{2^{-n\left(R_{10}+R_{20}\right)}}{(\ln 2) n}\right)}$. Finally, the bound of $P_{E_{6}}$ can be expressed as follows.

$P_{E_{6}} \leq 2^{-n\left[\Psi\left(\rho, P_{6}\right)-\rho\left(R_{12}+R_{10}+R_{13}+R_{23}\right)\right]}$, where,

$\Psi\left(\rho, P_{6}\right)=-\left(\alpha_{1} \log \left(q_{1}\right)+\alpha_{3} \log \left(q_{2}\right)+\frac{2^{-n\left(R_{10}+R_{20}\right)} \rho}{(\ln 2) n}\right)$

$q_{1}=\sum_{y_{1_{t}}}\left[\sum_{x_{11_{t}}} P\left(x_{11_{t}}\right)\left(P\left(y_{1_{t}} \mid x_{11_{t}}\right)\right)^{\frac{1}{1+\rho}}\right]^{1+\rho}$,

$q_{2}=\sum_{y_{3} t}\left[\sum_{x_{13_{t}}, x_{23_{t}}} P\left(x_{13_{t}}, x_{23_{t}}\right)\left(P\left(y_{3_{t}} \mid x_{13_{t}}, x_{23_{t}}\right)\right)^{\frac{1}{1+\rho}}\right]^{1+\rho}$.

Now, it can be easily verified that $\left.\Psi\left(\rho, P_{6}\right)\right|_{\rho=0}=0$. Also, it can be shown that:

$$
\begin{aligned}
\left.\frac{d \Psi\left(\rho, P_{6}\right)}{d \rho}\right|_{\rho=0}= & \alpha_{1} I\left(X_{11} ; Y_{1}\right)+\alpha_{3} I\left(X_{13}, X_{23} ; Y_{3}\right) \\
& -\frac{2^{-n\left(R_{10}+R_{20}\right)}}{(\ln 2) n}
\end{aligned}
$$

Hence, it can be easily noted that $P_{E_{6}} \rightarrow 0$ as $n \rightarrow \infty$ if:

$$
R_{12}+R_{10}+R_{20} \leq \alpha_{1} I\left(X_{11} ; Y_{1}\right)+\alpha_{3} I\left(X_{13}, X_{23} ; Y_{3}\right) .
$$

The other error events in this group can be analyzed similarly. Hence, $\left(P_{E_{5}}, P_{E_{6}}\right) \rightarrow 0$ as $n \rightarrow \infty$ if:

$$
\begin{aligned}
R_{21}+R_{10}+R_{20} \leq & \alpha_{2} I\left(X_{22} ; Y_{2}\right)+\alpha_{3} I\left(X_{13}, X_{23} ; Y_{3}\right) \\
R_{12}+R_{21}+R_{10}+R_{20} \leq & \alpha_{1} I\left(X_{11} ; Y_{1}\right)+\alpha_{2} I\left(X_{22} ; Y_{2}\right) \\
& +\alpha_{3} I\left(X_{13}, X_{23} ; Y_{3}\right) .
\end{aligned}
$$

The rate constraints obtained from the first error group are

$R_{10} \leq \alpha_{3} I\left(X_{13} ; Y_{3} \mid U_{3}, X_{23}\right), R_{20} \leq \alpha_{3} I\left(X_{23} ; Y_{3} \mid U_{3}, X_{13}\right)$,

$R_{10}+R_{20} \leq \alpha_{3} I\left(X_{13}, X_{23} ; Y_{3} \mid U_{3}\right)$. 
Finally, by applying the rate constraints in (43) and (44) into the Gaussian channel in (1) with the signaling in (3), we obtain the achievable rate region given in Theorem 1 . Then, we take the expectation to incorporate the randomness of the fading channel.

\section{APPENDIX B \\ Proof of the Occurrence Probability of CASE 1}

The probability of case 1 can be simply derived as follows. First since the Rayleigh distribution extends from 0 to $\infty$ and the channel parameters are independent, the occurrence probability can be expressed as

$$
\begin{aligned}
P\left[g_{12} \leq g_{10}, g_{21} \leq g_{20}\right] & =P\left[g_{12}^{2} \leq g_{10}^{2}, g_{21}^{2} \leq g_{20}^{2}\right] \\
& =P\left[g_{12}^{2} \leq g_{10}^{2}\right] P\left[g_{21}^{2} \leq g_{20}^{2}\right] .
\end{aligned}
$$

Then, $P\left[g_{12}^{2} \leq g_{10}^{2}\right]$ becomes

$$
\begin{aligned}
& P\left[g_{12}^{2} \leq \mu, g_{10}^{2}=\mu\right]=\int_{0}^{\infty} P\left(g_{12}^{2} \leq \mu\right) P\left(g_{10}^{2}=\mu\right) \partial \mu \\
& =\int_{0}^{\infty}\left(1-\exp \left\{\frac{-\mu}{\overline{g_{12}^{2}}}\right\}\right) \cdot \frac{1}{\overline{g_{10}^{2}}} \exp \left\{\frac{-\mu}{\overline{g_{10}^{2}}}\right\} \partial \mu=\frac{\mu_{12}}{\mu_{10}+\mu_{12}} .
\end{aligned}
$$

$P\left[g_{21}^{2} \leq g_{20}^{2}\right]$ can be obtained in a similar way. Then, $P\left[g_{12} \leq\right.$ $\left.g_{10}, g_{21} \leq g_{20}\right]$ is given as in (15).

\section{REFERENCES}

[1] A. Khandekar, N. Bhushan, J. Tingfang, and V. Vanghi, "LTE-advanced heterogeneous networks," in Proc. EW Conf., 2010, pp. 978-982.

[2] D. Gesbert et al., "Multi-cell MIMO cooperative networks: A new look at interference," IEEE J. Sel. Areas Commun., vol. 28, no. 9, pp. 1380-1408, Dec. 2010.

[3] L. Lei, Z. Zhong, C. Lin, and X. Shen, "Operator controlled deviceto-device communications in LTE-advanced networks," IEEE Wireless Commun., vol. 19, no. 3, pp. 96-104, Jun. 2012.

[4] M. J. Yang, S. Y. Lim, H. J. Park, and N. H. Park, "Solving the data overload: Device-to-device bearer control architecture for cellular data offloading," IEEE Veh. Technol. Mag., vol. 8, no. 1, pp. 31-39, Mar. 2013.

[5] N. Golrezaei, A. F. Molisch, A. G. Dimakis, and G. Caire, "Femtocaching and device-to-device collaboration: A new architecture for wireless video distribution," IEEE Commun. Mag., vol. 51, no. 4, pp. 142-149, Apr. 2013.

[6] M. Hasan, E. Hossain, and D. Niyato, "Random access for machineto-machine communication in LTE-advanced networks: Issues and approaches," IEEE Commun. Mag., vol. 51, no. 6, pp. 86-93, Jun. 2013.

[7] D. Astely et al., "LTE: The evolution of mobile broadband," IEEE Commun. Mag., vol. 47, no. 4, pp. 44-51, Apr. 2009.

[8] T. M. Cover and J. A. Thomas, Elements of Information Theory, 2nd ed. New York, NY, USA: Wiley, 2006.

[9] A. Sendonaris, E. Erkip, and B. Aazhang, "User cooperation diversity. Part I. System description," IEEE Trans. Commun., vol. 51, no. 11, pp. 1927-1938, Nov. 2003.

[10] S. Bakim and O. Kaya, "Cooperative strategies and achievable rates for two user OFDMA channels," IEEE Wireless Commun., vol. 10, no. 12, pp. 4029-4034, Dec. 2011.

[11] F. M. J. Willems, E. C. van der Meulen, and J. P. M. Schalkwijk, "Achievable rate region for the multiple-access channel with generalized feedback," in Proc. Annu. Allerton Conf. Commun., Control Comput., 1983, pp. 284-292.

[12] W. Mesbah and T. Davidson, "Optimized power allocation for pairwise cooperative multiple access," IEEE Trans. Signal Process., vol. 56, no. 7, pp. 2994-3008, Jul. 2008.

[13] L. Li, N. Jindal, and A. Goldsmith, "Outage capacities and optimal power allocation for fading multiple-access channels," IEEE Trans. Inf. Theory, vol. 51, no. 4, pp. 1326-1347, Apr. 2005.
[14] R. Narasimhan, "Individual outage rate regions for fading multiple access channels," in Proc. IEEE ISIT, Jun. 2007, pp. 1571-1575.

[15] L. Weng, A. Anastasopoulos, and S. S. Pradhan, "Diversity gain regions for MIMO fading broadcast channels," IEEE Trans. Commun., vol. 59, no. 10, pp. 2716-2728, Oct. 2011.

[16] L. Weng, S. S. Pradhan, and A. Anastasopoulos, "Error exponent regions for Gaussian broadcast and multiple-access channels," IEEE Trans. Commun., vol. 54, no. 7, pp. 2919-2942, Jul. 2008.

[17] J. Laneman, D. Tse, and G. Wornell, "Cooperative diversity in wireless networks: Efficient protocols and outage behavior," IEEE Trans. Inf. Theory, vol. 50, no. 12, pp. 3062-3080, Dec. 2004.

[18] M. O. Hasna and M. S. Alouini, "Optimal power allocation for relayed transmissions over Rayleigh fading channels," in Proc. IEEE VTC-Spring, Apr. 2003, pp. 2461-2465.

[19] A. Abu Al Haija and M. Vu, "Rate maximization for half-duplex multiple access with cooperating transmitters," IEEE Trans. Commun., vol. 61, no. 9, pp. 3620-3634, Sep. 2013.

[20] R. G. Gallager, Information Theory and Reliable Communication. New York, NY, USA: Wiley, 1968.

[21] A. Abu Al Haija and M. Vu, "A half-duplex cooperative scheme with partial decode-forward relaying," in Proc. IEEE ISIT, Aug. 2011, pp. $1886-1890$.

[22] A. Goldsmith, Wireless Communications, 1st ed. Cambridge, U.K.: Cambridge Univ. Press, 2005.

[23] A. Abu Al Haija and M. Vu, "Outage analysis for uplink mobile-tomobile cooperation," in Proc. IEEE GLOBECOM Workshop, Dec. 2013, pp. $579-584$

[24] R. El Gamal and Y.-H. Kim, Network Information Theory, 1st ed. Cambridge, U.K.: Cambridge Univ. Press, 2011.

[25] D. Slepian and J. Wolf, "A coding theorem for multiple access channels with correlated sources," Bell Syst. Tech. J., vol. 52, no. 7, pp. 1037-1076, Sep. 1973.

[26] R. Tandon and S. Ulukus, "Dependence balance based outer bounds for Gaussian networks with cooperation and feedback," IEEE Trans. Inf. Theory, vol. 57, no. 7, pp. 4063-4086, Jul. 2011.

[27] A. Hekstra and F. Willems, "Dependence balance bounds for single output two-way channels," IEEE Trans. Inf. Theory, vol. 35, no. 1, pp. 44-53, Jan. 1989.

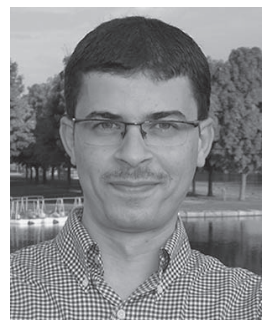

Ahmad Abu Al Haija (S'10) received the B.Sc. and M.Sc. degrees in electrical engineering from Jordan University of Science and Technology (JUST), Irbid, Jordan, in 2006 and 2009, respectively. He is currently working toward the Ph.D. degree at McGill University, Montreal, QC, Canada. Between February 2013 and August 2014, he was a Visiting Student with Tufts University, Medford, MA, USA. His research interest includes cooperation in multiuser channels, resource allocation for cooperative communication systems, wireless communications, and performance analysis and evaluation over fading channels.

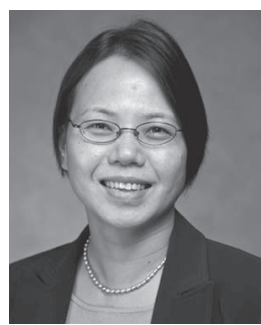

Mai Vu (M'06-SM'13) received the Bachelor's degree in computer systems engineering from RMIT University, Melbourne, Australia; the M.S.E. degree in electrical engineering from The University of Melbourne, Melbourne; and the Ph.D. degree in electrical engineering from Stanford University, Stanford, CA, USA. From 2006 to 2008, she worked as a Lecturer and a Researcher with the School of Engineering and Applied Sciences, Harvard University, Cambridge, MA, USA. From 2009 to 2012, she was an Assistant Professor of electrical and computer engineering, McGill University, Montreal, QC, USA. Since January 2013, she has been an Associate Professor with the Department of Electrical and Computer Engineering, Tufts University, Medford, MA, USA. Her research interests include wireless systems, signal processing, and networked communications. She has published extensively in the areas of cooperative and cognitive communications, relay networks, MIMO capacity and precoding, and energy-efficient communications. She has served on the technical program committee of numerous IEEE conferences and is currently an editor for the IEEE TRANSACTIONS ON WIRELESS COMMUNICATIONS. 